\title{
Don't Ditch the Laptop Just Yet: A Direct Replication of Mueller and Oppenheimer's (2014) Study 1 Plus Mini-Meta-Analyses Across Similar Studies
}

Heather L. Urry ${ }^{1}$, Chelsea S. Crittle ${ }^{1}$, Victoria A. Floerke ${ }^{1}$, Michael Z. Leonard ${ }^{1}$, Clinton S. Perry, III ${ }^{1}$, Naz Akdilek ${ }^{1}$, Erica R. Albert ${ }^{1}$, Avram J. Block ${ }^{1}$, Caroline Ackerley Bollinger ${ }^{1}$, Emily M. Bowers ${ }^{1}$, Renee S. Brody ${ }^{1}$, Kelly C. Burk ${ }^{1}$, Ally Burnstein ${ }^{1}$, Allissa K. Chan ${ }^{1}$, Petrina C. Chan ${ }^{1}$, Lena J. Chang ${ }^{1}$, Emily Chen ${ }^{1}$, Chakrapand Paul Chiarawongse $^{1}$, Gregory Chin ${ }^{1}$, Kathy Chin $^{1}$, Ben G. Cooper ${ }^{1}$, Katherine Corneilson ${ }^{1}$, Amanda M. Danielson ${ }^{1}$, Elizabeth S. Davis ${ }^{1}$, Ycar Devis ${ }^{1}$, Melissa Dong ${ }^{1}$, Elizabeth K. Dossett ${ }^{1}$, Nick Dulchin ${ }^{1}$, Vincent N. Duong ${ }^{1}$, Ben Ewing ${ }^{1}$, Julia Mansfield Fuller ${ }^{1}$, Thomas E. Gartman $^{1}$, Chad R. Goldberg ${ }^{1}$, Jesse Greenfield ${ }^{1}$, Selena Groh ${ }^{1}$, Ross A. Hamilton ${ }^{1}$, Will Hodge ${ }^{1}$, Dylan Van Hong ${ }^{1}$, Joshua E. Insler ${ }^{2}$, Aava B. Jahan ${ }^{1}$, Jessica Paola Jimbo ${ }^{1}$, Emma M. Kahn ${ }^{1}$, Daniel Knight ${ }^{1}$, Grace E. Konstantin ${ }^{1}$, Caitlin Kornick ${ }^{1}$, Zachary J. Kramer ${ }^{1}$, Meghan S. Lauz? ${ }^{1}$, Misha S. Linnehan ${ }^{1}$, Tommaso Lombardi ${ }^{1}$, Hayley Long ${ }^{1}$, Alec J. Lotstein ${ }^{1}$, Myrna-Nahisha A. Lyncee ${ }^{1}$, Monica Gabriella Lyons $^{1}$, Eli Maayan ${ }^{1}$, Nicole Marie May ${ }^{1}$, Elizabeth C. McCall ${ }^{1}$, Rhea Ann Charlotte Montgomery-Walsh ${ }^{1}$, Michael C. Morscher ${ }^{1}$, Amelia D. Moser $^{3}$, Alexandra S. Mueller ${ }^{1}$, Christin A. Mujica ${ }^{1}$, Elim $\mathrm{Na}^{4}$, Isabelle R. Newman ${ }^{1}$, Meghan K. O'Brien ${ }^{1}$, Katherine Alexandra Ochoa Castillo ${ }^{1}$, Zaenab Ayotola Onipede ${ }^{1}$, Danielle A. Pace ${ }^{1}$, Jasper Park $^{1}$, Angeliki Perdikari ${ }^{1}$, Catherine Perloff ${ }^{1}$, Rachel C. Perry ${ }^{1}$, Akash Pillai $^{1}$, Avni Rajpal ${ }^{1}$, Emma Ranalli ${ }^{1}$, Jillian E. Schreier ${ }^{1}$, Justin Shangguan ${ }^{1}$, Micaela Jen Silver ${ }^{1}$, Avery Glennon Spratt ${ }^{1}$, Rachel E. Stein ${ }^{1}$, Grant J. Steinhauer ${ }^{1}$, Devon K. Valera ${ }^{1}$, Samantha M. Vervoordt ${ }^{1}$, Lena Walton ${ }^{1}$, Noah W. Weinflash ${ }^{1}$, Karen Weinstock ${ }^{1}$, Jiaqi Yuan ${ }^{1}$, Dominique T. Zarrella ${ }^{1}$, \& Jonah Zarrow ${ }^{1}$

1 Tufts University

${ }^{2}$ Rush Medical College of Rush University Medical Center

${ }^{3}$ McLean Hospital

${ }^{4}$ Boston University School of Medicine

Preprint accepted for publication in Psychological Science on July 30, 2020. 


\begin{abstract}
In this direct replication of Mueller and Oppenheimer's (2014) Study 1, participants watched a lecture while taking notes with a laptop $(N=74)$ or longhand $(N=68)$. After brief distraction and without the opportunity to study, they took a quiz. Like the original study, laptop participants took notes containing more words spoken verbatim by the lecturer and more words overall than longhand participants. However, laptop participants did not perform better on the quiz than longhand participants. Exploratory meta-analyses of eight similar studies echoed this pattern. In addition, in both the original study and our replication, higher word count was associated with better quiz performance and higher verbatim overlap was associated with worse quiz performance, but the latter finding was not robust in our replication. Overall, results do not support the idea that longhand note-taking improves immediate learning via better encoding of information. Preregistration, materials, data, and code: https://osf.io/tr868/.

Keywords: note-taking, laptop, longhand
\end{abstract}

"Ditch the laptop and pick up a pen, class. Researchers say it's better for note taking."

—Elahe Izadi, August 26, 2014, Washington Post

In educational settings, students and professors alike are keen to facilitate student learning. One common strategy students adopt is to take notes during class using pen and paper or a laptop. Which note-taking medium promotes better learning? Mueller and Oppenheimer (2014) conducted a set of three experiments to find out.

In each experiment, participants watched a prerecorded lecture. Prior to watching the lecture, participants received either a laptop or pen and paper so they could take notes. They subsequently took a quiz about the lecture material. In two of three experiments, in which participants had no opportunity to study their notes, longhand note-taking resulted in better performance than laptop note-taking on items putatively tapping conceptual understanding. In a third experiment, the difference was found only amongst participants who studied their notes prior to taking the quiz a week later. In all three studies, participants took more notes in the laptop than longhand condition, and their notes included more of the words used by the lecturer in the laptop than longhand condition.

This work has been influential. For one, it may be guiding teaching decisions; it's frequently featured as a point of discussion amongst educators about the decision to allow or ban laptops in the classroom (one example: Holstead, 2015). Moreover, the work

This preprint was generated on Thu Jul 30 19:51:47 2020.

Correspondence concerning this article should be addressed to Heather L. Urry, 490 Boston Ave, Department of Psychology, Tufts University, Medford, MA, 02155, USA. E-mail: heather.urry@tufts.edu 
captured public imagination, with pieces published by the Washington Post, NPR, Scientific American and other outlets. It has inspired headlines suggesting that students should ditch their laptops and take notes by hand, as highlighted in the epigraph; otherwise, they perform worse. It has also captured academic imagination; as of July 12, 2020, Mueller and Oppenheimer (2014) have been cited more than 1,000 times (Google Scholar), and the paper's Attention score places it in "the top 5\% of all research outputs ever tracked by Altmetric" (over 15 million). It has also inspired several close replications (Kirkland, 2016; Luo, Kiewra, Flanigan, \& Peteranetz, 2018; Mitchell \& Zheng, 2017; Morehead, Dunlosky, \& Rawson, 2019).

In the current study $(N=142)$, we present our preregistered direct replication of Study 1 by Mueller and Oppenheimer $(2014 ; N=65)$. Participants took notes with a laptop or a pen while they watched one of five TED talks. After a distractor-filled delay, they took a quiz that assessed their grasp of the material. We measured quiz performance, the number of words in their notes, and verbatim overlap between their notes and words used by the lecturer

In confirmatory analyses, we tested the hypothesis that longhand note-taking would lead to better performance on conceptual quiz items than laptop note-taking. Such a result would indicate that note-taking medium impacts transfer of new information to long-term memory, an extension of Di Vesta and Gray's (1972) encoding hypothesis. We also tested the hypotheses that laptop note-taking would lead to more words in the notes (and, specifically, more words spoken verbatim by the lecturer) than longhand note-taking. Finally, we conducted exploratory mini-meta-analyses across similar studies to generate cumulative estimates of the size of note-taking effects on immediate quiz performance and notes contents to date.

\section{Method}

We report how we determined our sample size, and all data exclusions, manipulations, and measures in the study. This study was approved by the Social, Behavioral, and Educational Research Institutional Review Board at Tufts University. All participants provided written informed consent prior to participating.

We preregistered this study on March 7, 2017 (see https://osf.io/qe3wb/wiki/home/) Our materials, data, and analysis scripts are available on the Open Science Framework at https://osf.io/tr868/. When reporting results for the studies published by Mueller and Oppenheimer (2014), we relied on updated data files posted at https://osf.io/t43ua/ as part of their 2018 corrigendum.

\section{Participants}

We recruited participants through posts on social media, emails to acquaintances and outreach lists, and flyers in heavily trafficked locations on campus. Undergraduates interested in participating were directed to complete an online screening survey that confirmed 
they were a college student and at least 18 years old; eligible individuals were then redirected to a scheduling website. Our recruitment materials are available on p. 58-59 of the pdf at https://osf.io/y3ty8.

A total of $N=145$ undergraduate students from Tufts University participated in the experiment individually, typically with two experimenters. Two participants provided no responses to quiz items and condition assignment was unclear for a third thus we excluded these three observations, leaving us with $N=142$ for analysis. Notes were unavailable for two participants, thus analyses involving variables derived from notes (word count, verbatim overlap) have two fewer observations. We present our sample size rationale in the supplementary materials.

Participants were randomly assigned to view one of five lectures in either the laptop or longhand note-taking condition. Overall, there were 68 participants in the laptop condition (12 to 14 per lecture), and 74 in the longhand condition (13 to 18 per lecture). We, thus, had $80 \%$ power to detect a standardized effect of note-taking condition of Cohen's $d=+/-$ 0.47 or larger for quiz performance variables, and Cohen's $d+/-0.48$ or larger for notes variables (two-tailed alpha $=.05$ ). And we had $80 \%$ power to detect equivalence of the note-taking effect to zero within bounds of Cohen's $d=+/-0.49$ for quiz performance variables, and Cohen's $d=+/-0.49$ for notes variables (alpha $=.05$ ). Equivalence tests examine whether one can reject the presence of an effect as extreme or more so than one's equivalence bounds, ideally the smallest effect size of interest (Lakens, Scheel, \& Isager, 2018).

Participants from all four graduation years were represented; the majority were sophomores (12\% first-years, $49 \%$ sophomores, $23 \%$ juniors, and $17 \%$ seniors). With regard to gender, $62 \%$ identified as female and $37 \%$ as male; 1 person declined to report their gender. With regard to race/ethnicity, 5\% were African American or Black, 24\% were Asian, 58\% were Caucasian or White, $5 \%$ were Hispanic or Latino/a/x, and 7\% were Multiracial; 2 people declined to report their race/ethnicity. Participants received USD $\$ 15$ in compensation.

\section{Materials}

Lectures. The lectures for this study were the same TED talks used in the original study. They lasted approximately 15 minutes each. Links to their location on the ted.com website, from which the videos were streamed and transcripts obtained, are available in supplementary materials.

Quiz performance. Participants responded to open-ended quiz items from the original study for each of the five lectures. Per Mueller and Oppenheimer (2014), we divided items into two types, factual-recall and conceptual-application. The extent to which the quiz items reflect a valid distinction between factual versus conceptual understanding is unclear. However, we use the factual versus conceptual labels from the original study to facilitate comparison.

There were 5-7 items for factual-recall performance (e.g., "According to the speaker, what kinds of stressful tasks most reliably raise the level of cortisol (a stress-related hormone)?" and 3-5 items for conceptual-application performance (e.g., "Why are the negative 
outcomes the speaker discusses (social problems, life expectancy, etc.) correlated with economic status within countries, but not across countries?"), depending on which lecture the participant viewed.

A total of 12-15 raters scored participants' open-ended responses based on a standard scoring key. For details regarding scoring and interrater reliability, see our supplementary materials.

For both factual-recall and conceptual-application item types, interrater reliability was excellent for all lectures. The minimum and maximum intraclass correlations (ICCs) across lectures, respectively, were .98 and .99 for factual-recall, and .90 and .98 for conceptual-application. We calculated a total index score for each participant as the mean across raters separately for factual-recall and conceptual-application scales (maximum $=10)$. We then standardized these scores across lectures like the original authors; we also computed proportion correct.

Content of notes: Word count and verbatim overlap. For each participant, we determined the number of words in their lecture notes and the degree to which threeword chunks of text (trigrams) from a transcript of the lecture were present in those notes using the tidytext package (Silge \& Robinson, 2016) in R. We expressed verbatim overlap as a percentage: $100 * L / T$, where $\mathrm{L}=$ number of lecture trigrams in participant notes, and $\mathrm{T}=$ total number of trigrams in participant notes.

Distractor tasks. As a distraction after watching the video lectures, participants completed, in order, a typing test, a questionnaire, and a reading span task. Based on experimenter reports of typing test start times and reading span task end times, participants were distracted for 24.02 minutes on average across distractor tasks, 95\% CI [23.36, 24.69]. Distraction duration was similar for participants in the laptop, $M=23.73,95 \%$ CI $[22.8$, 24.66], and longhand conditions, $M=24.29,95 \%$ CI $[23.33,25.26], \Delta M=0.56,95 \%$ CI $[-0.77,1.89], t(136.00)=0.84, p=.403$. Thus, the distraction period was sufficient to build in an approximately 30-minute delay between lecture and quiz as in the original study, and it did not vary by note-taking condition. For detailed information about the distractor tasks, please see our supplementary materials.

\section{Design and Procedure}

We collected data in person in various locations on Tufts University's Medford campus. Before participants arrived, experimenters assembled the relevant forms and opened the Qualtrics survey that would be used to administer all study procedures, including random assignment of participants to conditions. Our Qualtrics survey template is available at https://osf.io/s5gfd.

We used a 2 (condition: laptop, longhand $) \times 5$ (lecture) between-subjects factorial design for this experiment. After providing written informed consent, experimenters provided each participant either a pen and paper or an experimenter-owned laptop on which to take notes. If the participant brought headphones to the session, the experimenter ensured that participants were wearing them and they were plugged in to the jack on a second computer, typically another experimenter-owned laptop, that would display the lecture. They then 
said, "You will now watch a lecture on this monitor. Please use your normal classroom note-taking strategy. We're interested in how information is actually recorded during class lectures." The experimenter ensured that the display screen was visible and then moved to an area of the room outside of the participant's line of sight.

When the video ended, the experimenter retrieved the note-taking laptop or pen and paper, and said "Now, we'd like for you to complete several tasks here on this computer. This part of the study will take about 30 minutes in total. Please let me know after you've finished each task." Participants then completed the distractor tasks, with the experimenter moving out of the participant's line of sight for each task. After completing the distractor tasks, participants completed the quiz for the lecture they had viewed earlier.

Finally participants responded to a number of self-report questions about their notetaking medium preferences and beliefs, described in supplementary materials. They also indicated with which gender and racial or ethnic group(s) they most identify. At this point, experimenters debriefed participants, collected information required to compensate them via PayPal, thanked, and excused them.

\section{Deviations from the Original Method}

Our replication of Study 1 differed from the original in the following ways:

1. We did not collect GPA or SAT score information from participants because this sensitive information is not critical to replicating the key findings of the original study. Because these variables were collected after the manipulations and measures of interest in the original study, their omission could not have affected our replication results.

2. We administered all manipulations and measures via a Qualtrics survey. The survey linked to other websites for the reading span and typing test distractors. Doing so facilitated our ability to collect the data in a standardized way for every participant and minimized the risk of data loss given the number of experimenters who collected the data. It is possible that this change could have affected the key results.

3. We added an open-ended question asking participants to tell us what they think the study is about. We administered this item after the manipulations and measures of interest; its inclusion could not have affected the key results.

4. We recruited college undergraduates at Tufts University in 2017 rather than Princeton University circa 2013. These are both selective private institutions thus the populations of interest are similar; nevertheless, it is possible that drawing from a different population or time could have affected the key results. For example, there could have been a difference in the frequency with which students typically take notes with a laptop versus longhand in the two studies.

5. In the original Study 1, participants completed the study in a classroom, generally in groups of two, and the video lecture was presented via a projector on a screen 
at the front of the room. We could not ensure that a classroom setting would always be accessible to our experimenters, and could not provide a standardized set of laptops to experimenters. As such, participants viewed the lecture on a monitor, typically a laptop owned by an experimenter. When available, participants wore headphones/earbuds to minimize distraction. In addition, participants took notes on a laptop that was owned by an experimenter, when applicable. We do not think using a laptop/headphone set-up is likely to have affected the key results of interest for our replication of Study 1 because the original authors adopted the same procedure in Study 2. Variation in settings and types of laptops used for note-taking and lecture-watching could, however, have introduced variability that affected key results.

6. Dr. Mueller kindly provided the two 5-minute distractor tasks used in the original Study 1 but these materials were not amenable to administration via Qualtrics. Thus, alongside the reading span task - one of the three original distractors in Study 1 - we administered a 5-minute typing test and the Need for Cognition Scale instead. These are the same distractors used by the original authors in Study 2. As such, we do not think this change is likely to have affected the key results of interest for our replication of Study 1.

\section{Confirmatory Data Analysis and Inference Criteria}

We analyzed our data using $\mathrm{R}$ ( $\mathrm{R}$ Core Team, 2020) and wrote this manuscript in $\mathrm{R}$ Markdown via RStudio 1.3.1056 (RStudio Team, 2020) to maximize reproducibility and minimize copy-paste errors when reporting results. The papaja (Aust \& Barth, 2018) and knitr (Xie, 2015) packages were instrumental to producing the formatted document. We used an alpha of .05 for null hypothesis significance testing.

In accordance with the original study and our preregistered analysis plan, we conducted independent samples t-tests to determine whether assignment to the laptop condition influenced word count and degree of verbatim overlap in the notes that participants took compared to the longhand condition. We also conducted mixed-effect analyses of variance in quiz performance with note-taking condition as a fixed effect and lecture as a random effect with a random slope for note-taking condition. The original authors used the UNIANOVA command in SPSS for these analyses; we conducted them using the afex package in R (Singmann, Bolker, Westfall, Aust, \& Ben-Shachar, 2019).

In addition, we examined whether the effect sizes in the present study were significantly different from those reported in the original study by conducting two one-sided tests using the TOSTER package (Lakens, 2017). For the factual and conceptual performance variables, we set the upper bound to the size of the original effect $(d=0.01$ and 0.34 , respectively), and the lower bound to -999 . For the word count and verbatim overlap variables, we set the lower equivalence bound to the size of the original effect $(d=-1.43$ and -0.94 , respectively), and the upper bound to +999 . These analyses amount to inferiority tests (Lakens et al., 2018).

We also examined whether the effect sizes in the present study were equivalent to $d$ $=0$ using TOSTER. In this case, we set equivalence bounds to $+/-0.49$ for the two quiz 
performance variables or $+/-0.49$ for the two notes variables. We selected these equivalence bounds because they yield $80 \%$ power to detect equivalence given the final sample size, an approach to recommended by Lakens (2017) as one way of defining the smallest effect size of interest.

A successful replication of results should yield a statistically significant effect of notetaking condition on number of words (laptop > longhand), verbatim overlap (laptop > longhand), and conceptual-application performance (longhand > laptop). In addition, replication effect sizes should be neither significantly different from the original effect sizes nor equivalent to 0 . For a list of deviations from our preregistered analysis plan, please see our supplementary materials.

\section{Results}

\section{Preliminary Analyses}

Summary Statistics. Table A1 in supplementary materials shows descriptive statistics for and correlations between measured variables in our replication.

Influential Observations. We identified potentially influential observations, i.e., observations that may have biased the effect of note-taking condition (longhand [.5] versus laptop [-.5]) on our four criterion variables, as described in supplementary materials. We repeated our confirmatory analyses after excluding these influential observations, as specified in our pre-registration (see https://osf.io/qe3wb/wiki/home/); their exclusion did not alter conclusions about experimental effects.

\section{Confirmatory Analyses}

Figure 1 shows all four primary dependent variables as a function of note-taking condition; plots were generated using the yarrr package (Phillips, 2017). The top row plots standardized quiz performance; factual-recall items on the left and conceptual-application items on the right. The bottom row plots notes content; word count on the left, verbatim overlap on the right.

Effect of Note-taking Condition on Quiz Performance. Table 1 shows the fixed effect of note-taking condition on quiz performance in the original study by Mueller and Oppenheimer (2014; Study 1) and the present replication study. We show results for standardized performance scores, as presented in the original study, as well as proportion correct, a more intuitive measure of performance. As shown, removing influential observations from the replication data had little effect on conclusions thus results below include all observations.

Consistent with the original study, the difference in factual-recall performance between the laptop and longhand conditions was not significant (see Figure 1, top left). Based on standardized scores, the original study yielded a near-zero effect slightly favoring better factual-recall performance in the longhand than laptop condition (Hedges' $g=0.01,95 \%$ CI $[-0.48,0.50])$. The present replication study effect was negligible in the opposite direction 


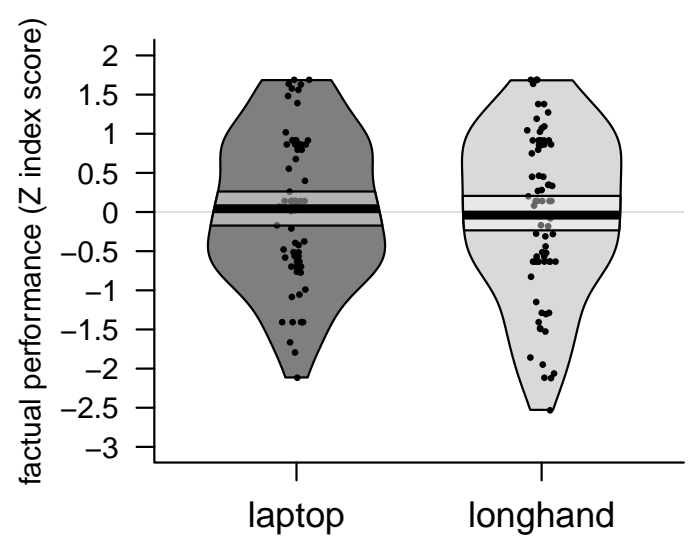

note-taking condition

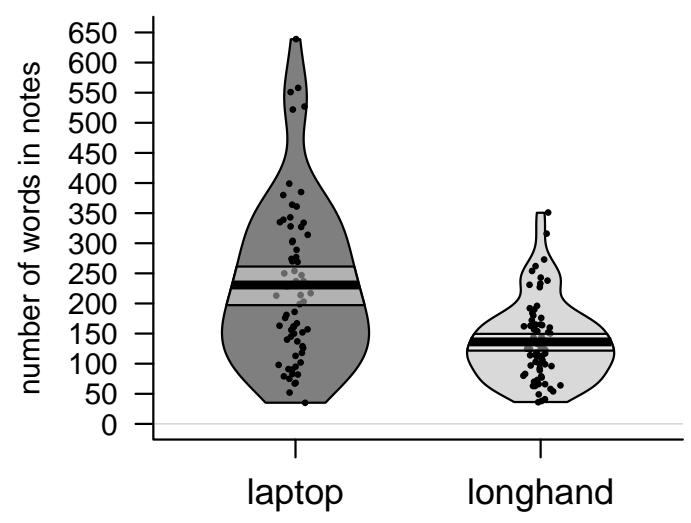

note-taking condition

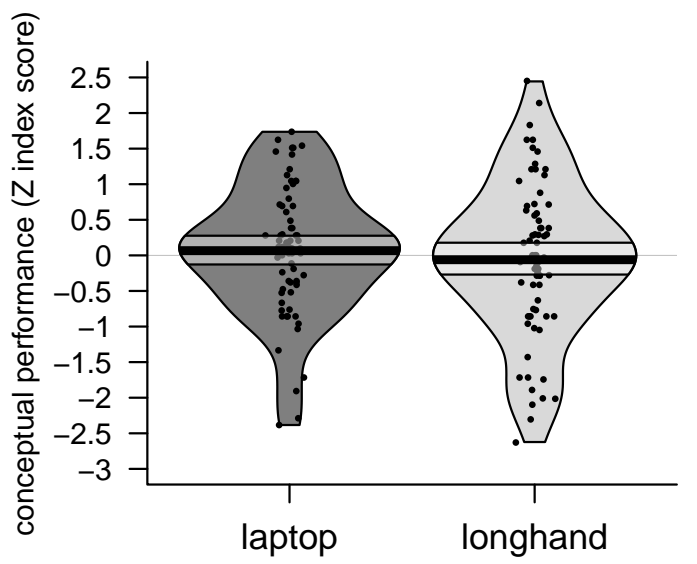

note-taking condition

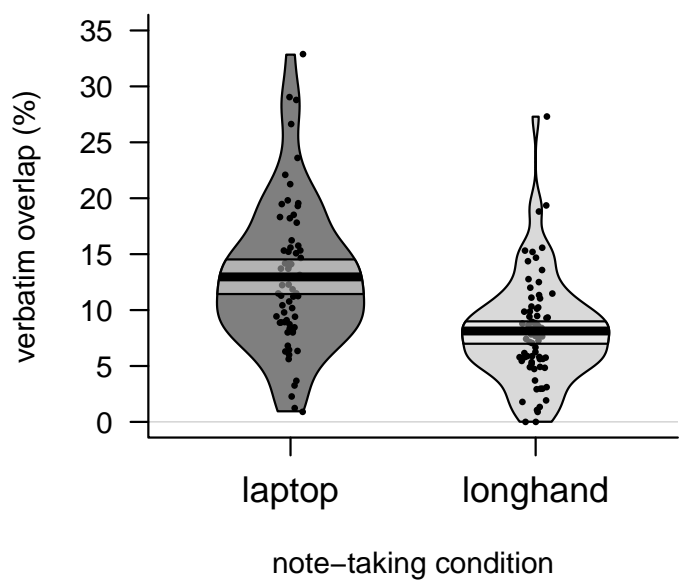

Figure 1. Violin plots depicting the four primary dependent variables by note-taking condition in the present replication study. For quiz performance (top row), we depict standardized scores. The mean is represented by the thick black line in each condition. Error bars, shown in lighter shading around mean, represent $95 \%$ confidence intervals. 
Table 1

Effect of note-taking condition on quiz performance (standardized and proportion correct scores) in the original study and in the present replication study.

\begin{tabular}{|c|c|c|c|c|c|c|}
\hline \multirow[b]{2}{*}{ measure } & \multicolumn{6}{|c|}{ ANOVA Results (Type III Sums of Squares) } \\
\hline & num Df & den Df & MSE & $\mathrm{F}$ & ges & $\operatorname{Pr}(>\mathrm{F})$ \\
\hline \multicolumn{7}{|l|}{ Factual-Recall (Z index score) } \\
\hline Original Study & 1 & 4 & 0.22 & 0.05 & .00 & .84 \\
\hline Replication Study - All observations & 1 & 4 & 0.09 & 0.13 & .00 & .74 \\
\hline Replication Study - Excluding influential & 1 & 4 & 0.07 & 0.00 & .00 & .98 \\
\hline \multicolumn{7}{|l|}{ Conceptual-Application (Z index score) } \\
\hline Original Study & 1 & 4 & 0.04 & 8.08 & .20 & .05 \\
\hline Replication Study - All observations & 1 & 4 & 0.08 & 0.59 & .02 & .48 \\
\hline Replication Study - Excluding influential & 1 & 4 & 0.03 & 2.68 & .05 & .18 \\
\hline \multicolumn{7}{|l|}{ Factual-Recall (proportion correct) } \\
\hline Original Study & 1 & 4 & 0.01 & 0.04 & .00 & .86 \\
\hline Replication Study - All observations & 1 & 4 & 0.00 & 0.07 & .00 & .81 \\
\hline Replication Study - Excluding influential & 1 & 4 & 0.00 & 0.02 & .00 & .91 \\
\hline \multicolumn{7}{|l|}{ Conceptual-Application (proportion correct) } \\
\hline Original Study & 1 & 4 & 0.00 & 9.40 & .34 & .04 \\
\hline Replication Study - All observations & 1 & 4 & 0.01 & 0.35 & .02 & .59 \\
\hline Replication Study - Excluding influential & 1 & 4 & 0.00 & 2.34 & .04 & .20 \\
\hline
\end{tabular}

Note. The fixed effects of condition on factual-recall and conceptual-application performance in the original Study 1 were $F(1,4.01)=0.046, p=.841$, and $F(1,4.09)=8.05, p=$ .046 , respectively, based on the UNIANOVA command in SPSS (see 2018 corrigendum files at https://osf.io/t43ua/). We report results from the corresponding analysis in $\mathrm{R}$ using the afex::aov_ 4 command. The values differ slightly due to differences in how SPSS and afex handle random effects, but substantive conclusions remain the same. ges = generalized eta-squared, a measure of effect size; $\operatorname{Pr}(>\mathrm{F})=p$ value

(Hedges' $g=-0.08,95 \% \mathrm{CI}[-0.41,0.25]$ ), not significantly different from the original effect, $t(139.96)=-0.55, p=.291$, and equivalent to $0+/-0.49, t(139.96)=2.45, p=.008$.

Contrary to the original study, the difference in conceptual-application performance was not significant (see Figure 1, top right). Based on standardized units, the original study yielded a small-medium effect suggesting better performance in the longhand than laptop condition (Hedges' $g=0.34,95 \% \mathrm{CI}[-0.16,0.83]$ ). The replication study effect was negligible in the opposite direction (Hedges' $g=-0.13,95 \% \mathrm{CI}[-0.45,0.20]$ ), significantly different from the original effect, $t(139.03)=-2.78, p=.003$, and equivalent to $0+/-0.49$, $t(139.03)=2.17, p=.016$.

In units of proportion correct, mean factual-recall performance in the laptop condition was .63 $(S D=.20,95 \%$ CI $[.58, .68])$; mean factual-recall performance in the longhand condition was .62 $(S D=.23,95 \%$ CI $[.57, .68])$. Mean conceptual-application performance 
in the laptop condition was $.74(S D=.19,95 \%$ CI $[.69, .78])$; mean conceptual-application performance in the longhand condition was .70 $(S D=.23,95 \%$ CI $[.65, .75])$.

Averaged across note-taking conditions, participants in the original study scored lower by a proportion of .05 on factual-recall items, $M=.58(S D=.21,95 \%$ CI $[.53, .63])$, than participants in this replication study, $M=.63(S D=.21,95 \%$ CI $[.59, .66])$. Similarly, participants in the original study scored lower by a proportion of .08 on conceptual-application items, $M=.63(S D=.23,95 \%$ CI $[.58, .69])$, than participants in this replication study, $M=.72(S D=.21,95 \%$ CI $[.68, .75])$.

Effect of Note-taking Condition on Content of Notes. Consistent with the original study, taking notes using a laptop, $M=230.69, S D=133.87,95 \%$ CI [198.03, 263.34], led to a higher word count than taking notes longhand, $M=136.16, S D=66.26$, $95 \%$ CI $[120.71,151.62], t(94.63)=-5.22, p<.001$ (see Figure 1, bottom left). Removing influential observations had little effect on the statistical results, $t(99.41)=-4.91, p<.001$. Both the original and replication studies yielded large effects suggesting a higher word count in the laptop than longhand condition (original Hedges' $g=-1.41,95 \%$ CI[-1.96, -0.86$]$, and replication Hedges' $g=-0.90,95 \%$ CI $[-1.25,-0.56])$. The replication study effect was significantly different from the original effect, $t(94.63)=3.02, p=.002$, but it was not equivalent to $0+/-0.49, t(94.63)=-2.34, p=.989$.

Again consistent with the original study, taking notes using a laptop, $M=12.97 \%$, $S D=6.53,95 \%$ CI $[11.37,14.56]$, led to more verbatim overlap with the lecture than writing notes longhand, $M=8.13 \%, S D=4.73,95 \%$ CI [7.03, 9.23], $t(119.46)=-4.98, p<.001$ (see Figure 1, bottom right). Removing influential observations had little effect on the statistical results, $t(119.30)=-5.58, p<.001$. Both studies yielded large effects suggesting a higher verbatim overlap in the laptop than longhand condition (original Hedges' $g=$ $-0.93,95 \% \mathrm{CI}[-1.44,-0.41]$, and replication Hedges' $g=-0.85,95 \% \mathrm{CI}[-1.19,-0.51])$. The replication study effect was not significantly different from the original effect, $t(119.46)=$ $0.45, p=.326$, nor was it equivalent to $0+/-0.49, t(119.46)=-2.08, p=.980$.

Averaged across note-taking conditions, participants in the original study typed 56.95 more words, $M=238.35$ ( $S D=116.79,95 \%$ CI $[209.41,267.29])$, than participants in this replication study, $M=181.40(S D=114.14,95 \%$ CI $[162.33,200.47])$. Levels of verbatim overlap were similar; participants in the original study exhibited just $1.09 \%$ greater verbatim overlap, $M=11.53 \%(S D=6.69,95 \%$ CI $[9.87,13.19])$, than participants in this replication, $M=10.44 \%(S D=6.14,95 \%$ CI $[9.42,11.47])$.

\section{Exploratory Mini-Meta-Analyses}

Our replication's experimental results are consistent with the original study by demonstrating a laptop superiority effect when it comes to the number of words in notes and extent of verbatim overlap with the lecture, and no effect of note-taking condition on factual quiz performance. However, our replication results are inconsistent with the original study by not demonstrating a longhand superiority effect when it comes to conceptual quiz performance. It may be that our particular instantiation resulted in false negative effects for conceptual items due to methodological differences (e.g., use of Qualtrics to collect the data, different 
population of undergraduates, variation in data collection settings, experimenters, and computer equipment). Thus, next we conducted exploratory mini-meta-analyses to integrate evidence across multiple similar studies as a more robust test of the hypothesis.

To estimate the effect of note-taking condition on quiz performance, word count, and verbatim overlap, we located a total of eight very similar studies that met the following criteria: 1) Experimentally manipulated laptop versus longhand note-taking, 2) Assessed immediate quiz performance on the same day as exposure to the lecture, 3) Used video lecture material, 4) Measured and reported results for quiz performance, word count, and verbatim overlap, and 5) Studied undergraduates. See our supplementary materials for information about our search strategy. Although eight studies is insufficient to make definitive conclusions, it does afford an interim aggregation of cumulative knowledge that can yield testable predictions for future work.

The set of $k=8$ studies was comprised of Study 1 and 2 by the original authors (Mueller \& Oppenheimer, 2014) (not Study 3, which assessed quiz performance one week later), two studies reported by Morehead et al. (2019) (immediate condition only), the current study, and three more single-study replications (Kirkland, 2016; Luo et al., 2018; Mitchell \& Zheng, 2017). We excluded participants in the laptop intervention condition in Mueller and Oppenheimer's Study 2 given that the goal of the intervention was to eliminate or reduce the difference between laptop and longhand conditions. Also, we could only metaanalyze $k=7$ studies for factual-recall and conceptual-application performance because the authors reported performance across item types in one study (Luo et al., 2018).

One source of variation across studies, despite otherwise similar methods, is the lecture video material. Morehead et al., Mitchell and Zheng, and the current replication used at least one of the original TED talk lectures; Kirkland and Luo et al. used other video material that lasted a bit longer than the original 15-minute videos (28 and 23 minutes, respectively). We excluded the recent study of 7th-9th-grade students (Frantz, Morling, \& Radu, 2018), in part because participants were not university undergraduates, which could introduce age-related heterogeneity, and in part because they do not report results for word count and verbatim overlap.

We ran five random effects meta-analyses to estimate the effect of note-taking condition on quiz performance (total, factual, conceptual) and the content of notes (word count, verbatim overlap) using the metafor package (Viechtbauer, 2010). We computed effect sizes as longhand minus laptop using the effsize (Torchiano, 2019) and compute.es (Re, 2013) packages.

The forest plot in Figure 2 summarizes quiz performance findings. Across studies, taking notes longhand versus with a laptop boosted total quiz performance across factual and conceptual item types to a negligible degree, Hedges' $g=0.04,95 \% \mathrm{CI}[-0.13,0.20]$. This effect was not statistically significant, $Z=0.46, p=.645$, and it also was equivalent to $0+/-0.38, Z=-4.10, p=<.001$. The equivalence bound of $d=0.38$ reflects the effect size the original study could detect with $33 \%$ power. Equivalence, thus, suggests that the meta-analytic effect size was too small to have been detected in the original study.

The effects for the two item types separately, factual-recall performance, Hedges' $g=$ 0.03, 95\% CI[-0.13, 0.19], and conceptual-application performance, Hedges' $g=0.14,95 \%$ 


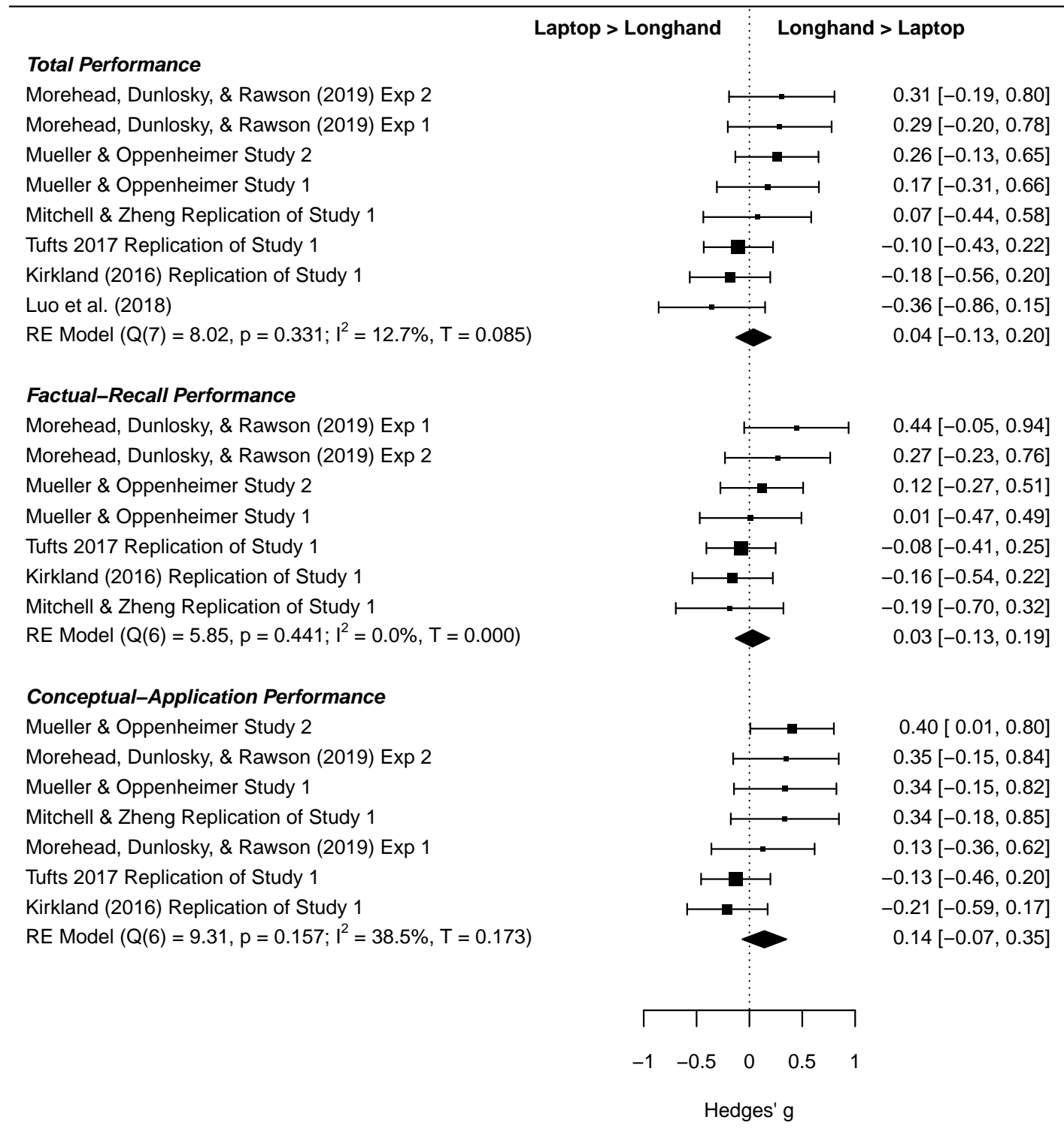

Figure 2. Effect sizes (standardized) for the quiz performance measures for the original study and all replications. For each measure, we present Hedges' $g$ point estimates in descending order. Error bars represent 95\% confidence intervals (CIs). The size of the symbols is inversely proportional to the variance of the estimate; larger symbols indicate more precise estimation. We generated overall estimates using a random effects (RE) model. Overall estimates are depicted with black diamonds. 


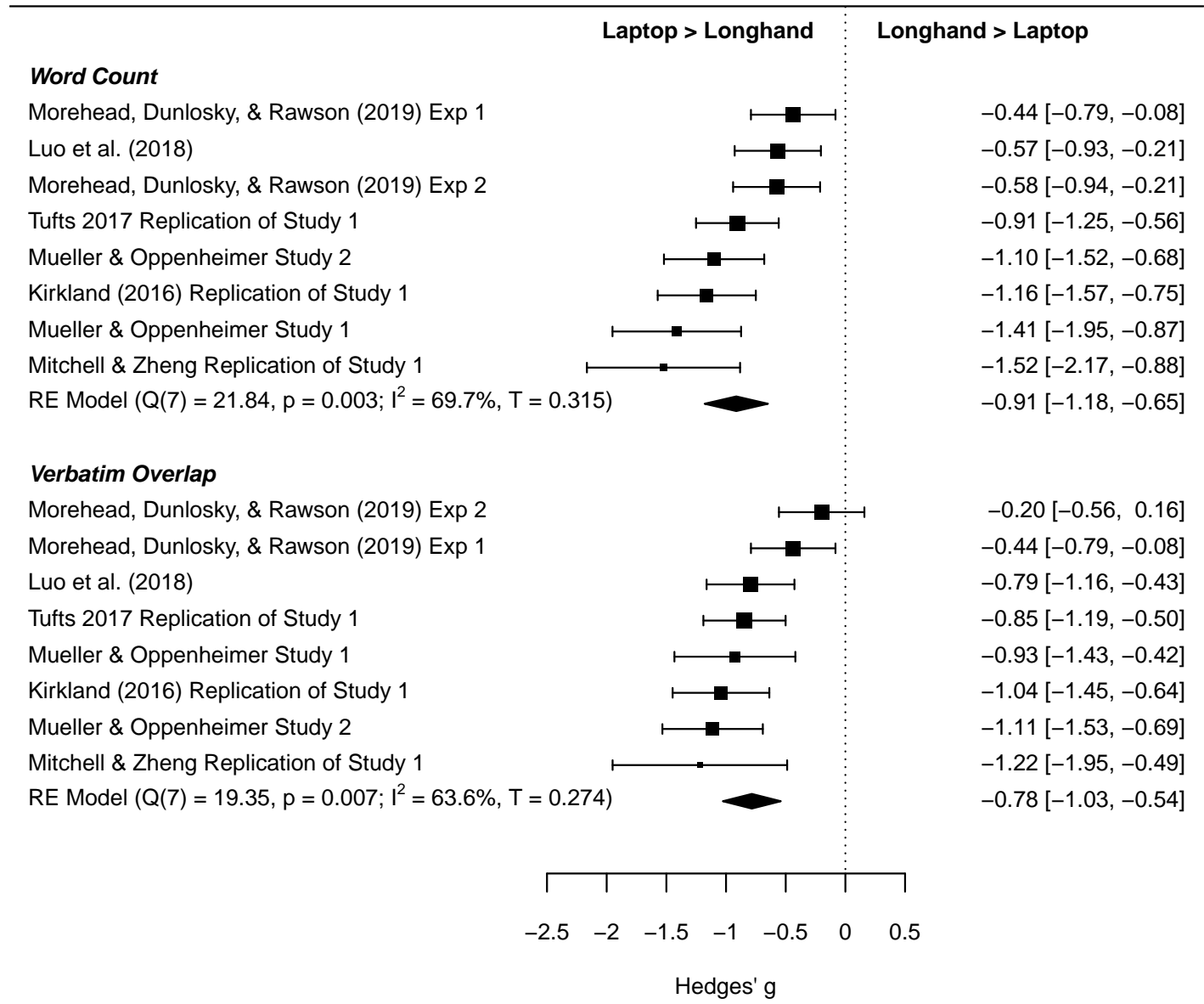

Figure 3. Effect sizes (standardized) for the notes content measures for the original study and all replications. For each measure, we present Hedges' $g$ point estimates in descending order. Error bars represent $95 \%$ confidence intervals (CIs). The size of the symbols is inversely proportional to the variance of the estimate; larger symbols indicate more precise estimation. We generated overall estimates using a random effects (RE) model. Overall estimates are depicted with black diamonds. 
CI [-0.07, 0.35], were negligible to very small. These effects were not statistically significant, $Z=0.36, p=.719$ and $Z=1.34, p=.182$, respectively, and both were equivalent to $0+/-$ $0.38, Z=-4.33, p=<.001$ and $Z=-2.27, p=.011$, respectively.

The forest plot in Figure 3 summarizes notes content findings. Consistent with the original study, taking notes with a laptop boosted both word count, Hedges' $g=-0.91,95 \%$ CI[-1.18, -0.65], and degree of verbatim overlap, Hedges' $g=-0.78,95 \% \mathrm{CI}[-1.03,-0.54]$, to a large degree. These effects were statistically significant, $Z=-6.75, p=<.001$ and $Z$ $=-6.34, p=<.001$, respectively, and neither of them were equivalent to $0+/-0.38, Z=$ $-3.92, p>.999$ and $Z=-3.24, p=.999$, respectively.

A modest percentage of the total variability across studies was due to heterogeneity of true effects for total quiz performance, $I^{2}=12.73 \%$. This appeared to be driven more so by conceptual-application performance, $I^{2}=38.54 \%$, than factual-recall performance, $I^{2}=.001 \%$. In terms of notes content, a large percentage of the total variability across studies was due to heterogeneity of true effects for word count, $I^{2}=69.69 \%$, and verbatim overlap, $I^{2}=63.58 \%$. Our supplementary materials address whether note-taking condition effects on notes content variables are correlated with note-taking condition effects on quiz performance variables at the study level.

\section{Additional Exploratory Analyses}

We present a number of additional exploratory analyses in supplementary materials which we summarize briefly here for the sake of completeness. In one set of exploratory analyses, we took a Bayesian approach to examine relative evidence for the replication and null hypotheses. Consistent with results presented above, results generally favored the replication hypothesis for notes variables and the null hypothesis for quiz performance variables.

In a second set of exploratory analyses, we conducted mixed-effect analyses of variance in quiz performance with item type treated as a factor (instead of examining factual and conceptual performance in separate analyses); there was no significant effect of note-taking condition either on its own or in interaction with item type; this was true in our replication and in the original study.

In a third set, we examined continuous predictors of quiz performance in linear mixedeffects regressions; such analyses could reveal hypothesized effects of note-taking condition by accounting for variance in quiz performance otherwise attributed to error in confirmatory analyses. However, there were no significant effects of note-taking condition when accounting for these extraneous variables. In line with the original study, higher word count was associated with better quiz performance; higher verbatim overlap was associated with worse quiz performance, but inconsistently so, depending on analysis.

In a fourth set, we examined laptop versus longhand note-taking preferences. Our replication participants were more likely to say they tended to take notes longhand; original study participants were more likely to say they tended to take notes using a laptop. Our replication participants also believed, on average, that taking notes longhand is better for 
learning; original study participants believed, on average, that there wasn't much of a difference.

\section{Discussion}

\section{Summary and Evaluation of Replication Results}

Mueller and Oppenheimer (2014) found in their first study that participants who took lecture notes on a laptop demonstrated poorer performance on putatively conceptual quiz items than their counterparts who took lecture notes by hand. Laptop notes contained more words and greater verbatim overlap with lecture content than longhand notes. Moreover, people whose notes had more words but less verbatim overlap performed better. Laptop versus lecture note-taking had no effect on factual-recall quiz performance.

In our replication study, laptop notes contained more words and greater verbatim overlap with lecture content than longhand notes. However, unlike the original study, we found only small, statistically nonsignificant differences in quiz performance as a function of note-taking medium. This conclusion was borne out in mixed-effect ANOVAS, equivalence tests, Bayesian analyses, and linear mixed-effect regressions. Thus, we replicated the experimental effect of note-taking condition assignment on notes but not quiz performance.

We also replicated correlational results reported by Mueller and Oppenheimer (2014). Consistent with the original study, we found that higher word count was associated with better quiz performance. We also found that higher verbatim overlap was associated with worse quiz performance, albeit less robustly. It would be tempting to conclude that taking more notes causes better quiz performance or that taking verbatim notes causes worse performance. However, we did not manipulate word count or the extent to which the notes exhibited verbatim overlap with the lecture thus alternative explanations are plausible. Higher word count or lower verbatim overlap may be third-variable proxies for motivation, conscientiousness, and/or interest, any of which might prompt students to take more notes in their own words and do better on the test.

\section{Mini-Meta-Analyses of Very Similar Studies}

There have been a number of parallel efforts by other researchers to replicate the experimental effect of note-taking condition on both quiz performance and notes content variables in undergraduates watching lecture videos. This is not surprising in light of the theoretical and practical importance of the findings.

Our mini-meta-analyses of studies that reported the same dependent measures in undergraduates - two in the original report plus six by independent researchers - suggested that the experimental effect on quiz performance was near zero irrespective of item type. Confidence intervals around the point estimates indicated that negligible to small effects favoring laptop or longhand superiority were both compatible with the data. There was modest heterogeneity in the extent to which this was true across studies. 
By contrast, across the board, these studies found that laptop note-taking boosted both word count and verbatim overlap with the lecture relative to longhand note-taking. Confidence intervals around the point estimates indicated that medium to large effects favoring laptop superiority were compatible with the data. However, there was considerable heterogeneity in the extent to which this was true across studies.

Our mini-meta-analyses, thus, replicated the experimental effect of note-taking condition on notes but not quiz performance. This reduces concern about the limitations of our single replication. However, because these meta-analyses included only eight studies, likely did not include all unpublished attempts to replicate the original study, and did not take into account publication bias, our meta-analytic estimates should be considered preliminary.

\section{Limitations and Future Directions}

Our direct replication of Mueller and Oppenheimer's (2014) Study 1 was limited by some deviations from the original study; we comment further on one, namely the nature of our data collection sessions. Specifically, $\sim 80$ students partnered to run data collection sessions on campus at various times of day outside of class. Many noted that sessions were subject to distractions and errors; sessions also varied in formality and equipment (i.e., laptops and headphones). Thus, situation noise was likely a considerable source of random error that could have reduced sensitivity to detect note-taking effects on quiz performance. Future studies examining laptop versus longhand note-taking effects should control the context to minimize these sources of random error.

There are several important directions for future research. First, we only considered the effect of laptop versus longhand note-taking on immediate testing with no opportunity to study. Some studies suggest that note-taking condition effects only occur when participants had the opportunity to study their notes (Luo et al., 2018; Mueller \& Oppenheimer, 2014, Study 3); future studies should focus experimental efforts in this direction.

Second, the studies in our meta-analyses mostly used TED talks as lectures. These are interesting and unfamiliar to students but also brief and unlike actual classroom lectures. Disallowing pauses to catch up on note-taking or ask questions takes the experimental context farther afield of reality. Future studies should use approaches that better represent real-world settings and new note-taking technologies (e.g., the eWriter examined by Morehead et al., 2019) and account for note-taking preferences. For example, our replication participants were more apt to say they generally took class notes by hand than original study participants. Maybe longhand note-taking has bigger effects on performance in people who typically take laptop notes. Although one study failed to observe a moderating effect of note-taking preference (Kirkland, 2016), higher-powered research is needed.

Third, future studies should, ideally, include a no-notes control condition to see the effect of taking notes regardless of medium (Jansen, Lakens, \& IJsselsteijn, 2017). Focusing on the laptop-longhand comparison without a no-notes control encourages dichotomous thinking when the story likely is more complicated. Jansen and colleagues (2017) suggest, for example, that a note-taking benefit "depends on the way lectures are presented, how notes are taken, and individual differences in cognitive abilities" (p. 231). 
Finally, the studies considered herein examined whether note-taking medium influences information encoding; they did not address other important issues that bear on the utility of laptops in classrooms. For example, laptops (and other web-enabled devices) can support active learning, and are necessary for learning for some disabled students; they may also be a source of distraction. Future studies must address these other issues.

\section{Psychological Science in the Classroom}

Psychologists have spearheaded several large-scale replication efforts like Reproducibility Project: Psychology (RP:P; Aarts et al., 2015) and "Many Labs" (e.g., Klein et al., 2014). Large-scale efforts alone, however, are insufficient to increase the frequency of replications; conducting "didactic replications" in our classes - like we did here - is another option (Frank \& Saxe, 2012; Gernsbacher, 2018; Grahe et al., 2012; Hawkins et al., 2018). Frank and Saxe (2012) argue, for example, that students in research methods courses often must conceive, design, and conduct studies in just a few weeks, often with little enthusiasm. Mentoring publication-worthy replication studies instead may simultaneously inspire curiosity and motivation in students and generate value outside the classroom. Mechanisms such as the Collaborative Replications and Education Project (Wagge et al., 2019) can support these efforts.

\section{Conclusion}

Our direct replication of Mueller and Oppenheimer's (2014) Study 1 showed that, relative to longhand note-taking, laptop note-taking boosted word count and verbatim overlap with lecture content, but it did not reduce knowledge of the lecture material after a brief delay with no opportunity to study. Results, thus, did not support the idea that longhand note-taking improves performance via better encoding of information.

When original and replication studies find different results, there are three interpretations: 1) there was a problem with the replication; 2) there was a problem with the original research; and 3) the phenomenon under study is not enduring or universal (i.e., there's a constraint on generality). These interpretations are not mutually exclusive. In fact, all three apply here. Situation noise was a problem with our replication. Weak evidence (large $\mathrm{p}$ value, Bayesian evidence favoring the null hypothesis) and a small sample size were problems with the original study. And a difference in note-taking medium preferences between the two may represent a constraint on generality.

Meta-analytic work can help to distill the conclusions we should draw from a body of studies. Our exploratory mini-meta-analyses of studies that used similar same-day laboratory experimental procedures failed to support longhand superiority for retention of lecture material. A recent meta-analysis across a larger, more heterogeneous set of classroom studies revealed a small effect that supported longhand superiority (Allen, LeFebvre, LeFebvre, $\&$ Bourhis, 2020). Neither of these meta-analyses considered the effect of publication bias or the extent to which the opportunity to study the notes or note-taking medium preference moderates findings. 
Until future research determines whether and when note-taking media influence academic performance, we conclude that students and professors who are concerned about detrimental effects of computer note-taking on encoding information to be learned in lectures may not need to ditch the laptop just yet. However, there's more work to be done using methods that more closely mimic actual educational contexts and that evaluates the impact of changing note-taking preferences. 


\section{Author Contributions}

We conducted this study as part of an undergraduate experimental psychology course (PSY 32, Experimental Psychology) at Tufts University in the Spring 2017 semester. The first author, H.L.U., is the professor who taught the course. The next four authors, C.S.C., V.A.F., M.Z.L., and C.S.P., were graduate student members of the teaching team, listed alphabetically. The remaining authors were undergraduate students in the course, listed alphabetically. All authors contributed to the design of the study. The undergraduate authors collected the data, analyzed it, and wrote their own empirical report in partial fulfillment of course requirements. The graduate authors facilitated the research in weekly lab sections. Formal contributions to this work according to the CRediT system (https://casrai.org/credit) were as follows: Conceptualization, all contributors; Data curation, H.L.U.; Formal analysis, H.L.U.; Funding acquisition, H.L.U.; Investigation, all undergraduate contributors; Methodology, H.L.U., C.S.C., V.A.F., M.Z.L., and C.S.P.; Project administration, H.L.U., C.S.C., V.A.F., M.Z.L., and C.S.P.; Resources, all contributors; Supervision, H.L.U., C.S.C., V.A.F., M.Z.L., and C.S.P.; Validation, H.L.U.; Visualization, H.L.U.; Writing - original draft, H.L.U. (this manuscript); Writing - review \& editing, H.L.U., V.A.F, C.S.C., R.S.R., J.P.J., E.M.K., M.S.L., M.G.L., A.D.M., C.A.M., S.M.V., and D.T.Z.; A number of undergraduate student authors could not be reached for inclusion in the review and editing process and to approve the final manuscript for submission; they are not, thus, listed as authors on this version of the manuscript.

\section{Acknowledgements}

We are grateful to Morton Ann Gernsbacher for discussion and feedback on an earlier version of this manuscript, and for a Faculty Research Awards Committee grant from Tufts University. 


\section{References}

Aarts, A., Anderson, J., Anderson, C., Attridge, P., Attwood, A., Axt, J., ..... (2015). Estimating the reproducibility of psychological science. Science, 349 (6251).

Allen, M., LeFebvre, L., LeFebvre, L., \& Bourhis, J. (2020). Is the pencil mightier than the keyboard? A meta-analysis comparing the method of notetaking outcomes. Southern Communication Journal, 1-12.

Aust, F., \& Barth, M. (2018). papaja: Create APA manuscripts with $R$ Markdown. Retrieved from https://github.com/crsh/papaja

Di Vesta, F. J., \& Gray, G. S. (1972). Listening and note taking. Journal of Educational Psychology, 63(1), 8.

Frank, M. C., \& Saxe, R. (2012). Teaching replication. Perspectives on Psychological Science, 7(6), 600-604.

Frantz, Z., Morling, B., \& Radu, N. (2018). Conceptual replication of Mueller and Oppenheimer (2014). PsyArXiv. https://doi.org/10.31234/osf.io/gkjzs

Gernsbacher, M. A. (2018). Three ways to make replication mainstream. Behavioral and Brain Sciences, 41(e129). https://doi.org/10.1017/S0140525X1800064X

Grahe, J. E., Reifman, A., Hermann, A. D., Walker, M., Oleson, K. C., Nario-Redmond, M., \& Wiebe, R. P. (2012). Harnessing the undiscovered resource of student research projects. Perspectives on Psychological Science, 7(6), 605-607.

Hawkins, R. X. D., Smith, E. N., Au, C., Arias, J. M., Catapano, R., Hermann, E., ... Frank, M. C. (2018). Improving the replicability of psychological science through pedagogy. Advances in Methods and Practices in Psychological Science, 1(1), 7-18. https://doi.org/10.1177/2515245917740427

Holstead, C. E. (2015). The benefits of no-tech note taking. The Chronicle of Higher Education. Retrieved from https://www.chronicle.com/article/The-Benefits-of-No-TechNote/228089

Jansen, R. S., Lakens, D., \& IJsselsteijn, W. A. (2017). An integrative review of the cognitive costs and benefits of note-taking. Educational Research Review, 22, 223-233.

Kirkland, K. M. (2016). The effect of note taking media and preference on the cognitive processes involved in learning. Undergraduate Honors Theses. Retrieved from https: //scholar.colorado.edu/honr_theses/1244

Klein, R. A., Ratliff, K. A., Vianello, M., Adams Jr., R. B., Bahník, Š., Bernstein, M. J., ... Nosek, B. A. (2014). Investigating variation in replicability: A "Many Labs" replication project. Social Psychology, 45(3), 142-152.

Lakens, D. (2017). Equivalence tests: A practical primer for t-tests, correlations, and metaanalyses. Social Psychological and Personality Science, 1, 1-8. https://doi.org/10.1177/ 1948550617697177 
Lakens, D., Scheel, A. M., \& Isager, P. M. (2018). Equivalence testing for psychological research: A tutorial. Advances in Methods and Practices in Psychological Science, 1(2), 259-269.

Luo, L., Kiewra, K. A., Flanigan, A. E., \& Peteranetz, M. S. (2018). Laptop versus longhand note taking: Effects on lecture notes and achievement. Instructional Science, 1-25.

Mitchell, A., \& Zheng, L. (2017). Examining longhand vs. Laptop debate: Evidence from a replication. Americas Conference on Information Systems (AMCIS) Proceedings.

Morehead, K., Dunlosky, J., \& Rawson, K. A. (2019). How much mightier is the pen than the keyboard for note-taking? A replication and extension of Mueller and Oppenheimer (2014). Educational Psychology Review, 1-28. https://doi.org/10.1007/s10648019-09468-2

Mueller, P. A., \& Oppenheimer, D. M. (2014). The pen is mightier than the keyboard: Advantages of longhand over laptop note taking. Psychological Science, 25(6), 11591168.

Phillips, N. (2017). Yarrr: A companion to the e-book "YaRrr!: The Pirate's Guide to R". Retrieved from https://CRAN.R-project.org/package=yarrr

R Core Team. (2020). R: A language and environment for statistical computing. Vienna, Austria: R Foundation for Statistical Computing. Retrieved from https://www.Rproject.org/

Re, A. C. D. (2013). Compute.es: Compute effect sizes. $R$ Package. Retrieved from http://cran.r-project.org/web/packages/compute.es

RStudio Team. (2020). RStudio: Integrated development environment for $r$. Boston, MA: RStudio, PBC. Retrieved from http://www.rstudio.com/

Silge, J., \& Robinson, D. (2016). Tidytext: Text mining and analysis using tidy data principles in R. JOSS, 1 (3). https://doi.org/10.21105/joss.00037

Singmann, H., Bolker, B., Westfall, J., Aust, F., \& Ben-Shachar, M. S. (2019). Afex: Analysis of factorial experiments. Retrieved from https://CRAN.R-project.org/package=afex

Torchiano, M. (2019). Effsize: Efficient effect size computation. https://doi.org/10.5281/ zenodo. 1480624

Viechtbauer, W. (2010). Conducting meta-analyses in R with the metafor package. Journal of Statistical Software, 36(3), 1-48. Retrieved from http://www.jstatsoft.org/v36/i03/

Wagge, J. R., Brandt, M. J., Lazarevic, L. B., Legate, N., Christopherson, C., Wiggins, B., \& Grahe, J. E. (2019). Publishing research with undergraduate students via replication work: The collaborative replications and education project. Frontiers in Psychology, 10 (247), 1-24. https://doi.org/10.3389/fpsyg.2019.00247

Xie, Y. (2015). Dynamic documents with $R$ and knitr (2nd ed.). Boca Raton, Florida: Chapman; Hall/CRC. Retrieved from https://yihui.name/knitr/ 


\author{
Supplementary Material
}

\title{
Sample Size Rationale
}

As noted in our preregistration, we planned to recruit as many participants as possible up to $N=250$ within the time available in the Spring 2017 semester. At a minimum, we sought to recruit $N=67$ participants, the number of participants in the original study. Ideally, we hoped to recruit at least $N=200$ participants. This would have yielded $80 \%$ statistical power to detect a condition effect as small as Cohen's $f=0.20$, which is equivalent to $\eta^{2}=.04$ or $d=0.40$. However, we ran out of time to reach this ideal target. Our sample of $\mathrm{N}=142$ more than doubles the original sample size and, thus, gives us greater power to detect effects of any size. That said, it is insufficient to detect effects smaller than Cohen's $d=0.47$ for quiz performance variables or Cohen's $d=0.48$ for notes variables.

\section{Lecture Videos}

The lecture videos we used in the replication at Tufts University in Spring 2017 were as follows:

- Mustafa Akyol - Faith versus Tradition in Islam

- Richard Wilkinson - How Economic Inequality Harms Societies

- Matt Ridley - When Ideas Have Sex

- Rajesh Rao - Computing a Rosetta Stone for the Indus Script

- Kevin Slavin - How Algorithms Shape Our World

These are the same videos used in the original Study 1.

\section{Quiz Scoring}

Each rater scored data for only one of the five lecture conditions blind to note-taking condition using one of the scoring keys available at https://osf.io/s5gfd/. Note that we reworded one quiz item after preregistration. Specifically, the original wording of one of the factual-recall items was, "What word/sound do researchers think this symbol might represent?" (from the "Computing a Rosetta Stone for the Indus" lecture). We did not see a symbol in the scoring document posted by the original authors. Based on the image shown in the TED talk, we revised the wording to say, "What word/sound do researchers think the fish-like symbol might represent?" Answers accepted as correct were star or meen.

Per the 2018 corrigendum to the original study, we converted the original scores to index scores. The corrigendum says that "a perfect score would be 1 point per question; 10 points total" (p. 1) but not how instances of partial credit were handled. We assigned values of 0 (for items for which the participant earned zero credit) and 1 (for items for which the participant earned partial or full credit). We calculated a total index score across items for each participant for each rater separately for factual-recall and conceptual-application 
scales. We then examined interrater reliability by computing intraclass correlations (ICC) for these total scores. Because a random sample of $\mathrm{k}=12-15$ raters scored the quiz for each participant and our intention was to average across raters as our measures of factual-recall and conceptual-application performance, we calculated the average measure ICC; this is $\operatorname{ICC}(2, \mathrm{k})$ according to Shrout and Fleiss (1979).

\section{Details about Distractor Tasks}

The typing test lasted 5 minutes (https://www.typing.com/student/test/5). Experimenters recorded two scores, number of words per minute and accuracy. (Instead of the typing test and questionnaire, the original Study 1 used two 5-minute tasks that were not amenable to administration via Qualtrics; see Deviations from the Original Method section in the main manuscript)

Participants also completed the Need for Cognition questionnaire (Petty, Cacioppo, \& Kao, 1984), indicating to what extent they agree or disagree that 18 statements are characteristic of them on a scale ranging from +4 , very strong agreement, to 0 , neither agreement nor disagreement, to -4, very strong disagreement. Items included "I would prefer complex to simple problems", "I try to anticipate and avoid situations where there is likely a chance I will have to think in depth about something," and "I find satisfaction in deliberating hard and for long hours." Scores were computed by summing after reversescoring 9 items, with mean substitution for two participants with one missing item. (We did not compute a score if there was more than one missing item; there were two participants missing all items and one missing eight items.) The range of possible values is -72 to 72 . Internal consistency reliability was acceptable in the present sample (hierarchical omega = $.84,95 \%$ CI[.79, .89], Cronbach's alpha $=.85,95 \%$ CI[.80, .89]).

Finally, participants completed an automated reading span task programmed in Inquisit (Millisecond.com; see an example and details at https://www.millisecond.com/download/library/rspan/). Participants saw a sequence of 3-7 letters that they were meant to hold in mind while also processing the meaning of sentences. Timing information provided by experimenters suggested that all but one participant did the reading span task for at least some period of time but only two participants completed the task in full because it would otherwise have taken longer than the time available. We have useable reading span task data from a total of 96 participants, as explained below. On average, these participants spent 15.36 minutes doing the reading span task, 95\% CI [14.91, 15.82].

Of the 46 participants for whom we do not have useable reading span task data, 20 were present in the Inquisit participant activity log; this suggested that the reading span task was administered but got discontinued in a way that failed to save the data. The remaining 26 participants were not present in the activity log; for these participants, experimenter notes for 6 pointed to technical difficulties. In one case, experimenters noted that they did not run the reading span task due to a problem with security settings. In five other cases, experimenters noted that they had to cut the task short, that they hit ctrl-q to leave the task, that they experienced undescribed technical difficulties, that they had to open the reading span task on a different laptop when the link in the Qualtrics 
survey did not work, or that they had to open the Qualtrics survey again which generated a new subject identification number. We have no information about why the remaining 20 participants were not present in the Inquisit participant activity log.

\section{Additional Self-Report Questions}

We asked participants a number of self-report questions after completing the quiz. For one, they rated "How much knowledge related to the topic of the talk did you have before today?" on a scale from 0, Not at all, to 5, Expert. We also asked them what year they'll graduate and their concentration, and gave them an open-ended prompt, "Do you normally take notes in class on your laptop or in a notebook? Why?" After that, they rated, "In general, do you think it is better for learning purposes to take notes on a laptop or in a notebook?" on a scale from 1, Laptop significantly better, to 9, Notebook significantly better. They also responded to four open-ended questions, "Does your choice to take notes on a laptop or in a notebook differ depending on whether it is a humanities, science, or math course?", "How long do you normally spend reviewing notes when studying for a test?", "Do you have any other thoughts regarding notetaking on a laptop vs. in a notebook?", and "What do you think this study is about?"

\section{Deviations from our Preregistration}

Our replication of Study 1 deviated from our preregistration in the following ways:

1. Our preregistration said we would use a $\mathrm{Z}$ test to compare the replication effect sizes to the original effect sizes; the one-sided tests are, rather, t-tests.

2. Our preregistration indicated we'd set lower and upper equivalence bounds of $+/$ Cohen's $d=.40$, or whatever effect size is detectable with $80 \%$ power given the final sample size. We went with the latter because, although we would have preferred to set narrower equivalence bounds, doing so would have required $n=107$ participants per group to have $80 \%$ power.

3. Our sole focus on trigrams as the indicator of verbatim overlap represents a deviation from our preregistered analysis plan. We originally planned to also examine the degree to which one- and two-word chunks of text reflected overlap but chose not to do so for the sake of simplicity. The original report found similar results for all three overlap measures, thus this deviation should not hamper the conclusions we draw.

\section{Summary Statistics for Measured Variables}

Table A1 shows descriptive statistics for and correlations between measured variables, computed in part using the psych (Revelle, 2018) and userfriendlyscience (Peters, 2017) packages. The four primary measured variables were factual-recall performance, conceptual-application performance, word count, and verbatim overlap. For quiz performance, we present standardized scores (referenced as factual $\mathrm{Z}$ and conceptual $\mathrm{Z}$ in the 
table) and proportion correct (referenced as factual proportion correct and conceptual proportion correct in the table) for both item types. Measured variables of secondary interest were need for cognition, knowledge related to the lecture, preference for taking notes with a laptop or in a notebook, typing speed and accuracy, and distraction duration.

Higher word count was modestly positively correlated with two of the four quiz performance variables (factual $\mathrm{Z}$ and conceptual proportion correct); higher verbatim overlap was modestly negatively correlated with two of the four quiz performance variables (factual $\mathrm{Z}$ and factual proportion correct). Higher factual-recall performance was modestly positively correlated with higher conceptual-application performance. Higher word count was strongly positively correlated with greater verbatim overlap. Longer distraction time was associated with lower conceptual-application performance (proportion correct only), rating longhand note taking as better, and slower typing speed. The remaining measured variables of secondary interest exhibited a similar pattern of very small correlations with quiz performance. Faster typing speed was correlated with higher word count and greater verbatim overlap. Higher typing accuracy was correlated with greater verbatim overlap and faster typing speed. 
Table A1

Descriptive statistics for and zero-order correlations between measured variables.

2. conceptual Z

$\begin{array}{rr}M & S D \\ 0.00 & 1.00\end{array}$

$$
95 \% C I
$$

$\begin{array}{lll}1 & 2 & 3\end{array}$

$[-0.17,0.17]$

$[-0.17,0.17]$

$.26^{* *}$

3. factual proportion correct

5. word count

$.63 \quad .21$

7. need for cognition

8. related knowledge

9. better laptop or notebook

10. typing speed (WPM)

$.72 \quad .21$

$[.59, .66]$

$[.68, .75]$

$.92^{* *}$

$\begin{array}{llllll}181.40 & 114.14 & {[162.33,200.47]} & .20^{*} & .14 & .14\end{array}$

$\begin{array}{lllllll}10.44 & 6.14 & {[9.42,11.47]} & -.17^{*} & -.02 & -.20^{*} & .01\end{array}$

$22.86 \quad 16.65$

$1.11 \quad 1.13$

$5.24 \quad 1.53$

$65.16 \quad 15.81$

$97.31 \quad 1.23$

$[20.06,25.65]$

$[0.92,1.29]$

$[4.99,5.49]$

$[62.5,67.82]$

$\begin{array}{lll}24.02 & 3.95 & {[23.36,24.69}\end{array}$

.01

.07

.04

.01

.03

11. typing accuracy $(\%)$

$\begin{array}{llll}{[23.36,24.69]} & -.04 & -.11 & .03\end{array}$

$.06-.08-.06$

$\begin{array}{lllll}.06 & -.13 & -.05 & .05 & .08\end{array}$

12. distraction duration

Note. * $\mathrm{p}<0.05 ; * * \mathrm{p}<0.01$;

$-.20^{*}$

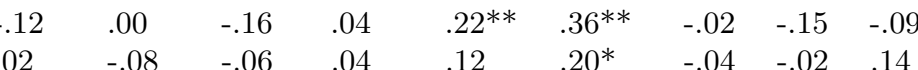

$.38^{* *}$

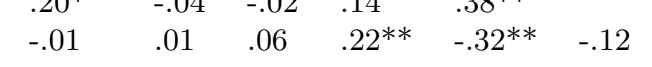
captured in the the "word count" and "verbatim overlap" entries. Higher scores reflect higher need for cognition ("NFC"), greater selfreported knowledge of the topic of the lecture ("related knowledge"), and greater belief that learning is better when notes are taken in a notebook rather than using a latop ("better laptop or notebook"). Z = standardized score; WPM = words per minute 


\section{Identifying Influential Observations}

To identify influential observations for each of our four dependent variables of primary interest, we computed Cook's Distance for each participant based on a set of four linear regression models examining note-taking condition as a predictor of each. Influential observations were defined as those for which Cook's Distance was greater than or equal to 4 times the mean Cook's Distance across all participants.

A total of 4 and 8 observations were identified for factual-recall and conceptualapplication performance, respectively. For factual-recall performance, there were 1 in the laptop condition, and 3 in the longhand condition. For conceptual-application performance, there were 3 in the laptop condition, and 5 in the longhand condition. In addition, a total of 6 and 7 observations were identified for word count and verbatim overlap, respectively. For word count, there were 5 in the laptop condition, and 1 in the longhand condition. For verbatim overlap, there were 6 in the laptop condition, and 1 in the longhand condition.

We repeated our confirmatory analyses without these influential observations included, as specified in our pre-registration (see https://osf.io/qe3wb/wiki/home/). Their exclusion did not alter conclusions.

\section{Literature Search Strategy for the Mini-Meta-Analyses}

To locate studies to include in our exploratory mini-meta-analyses, we used the Google and Google Scholar databases to search for articles that were methodologically similar to the original work by Mueller and Oppenheimer (2014). In our search, we used terms such as: notes, note taking, laptop, longhand, and academic performance. We also used Google Scholar to see if any articles that cited Mueller and Oppenheimer (2014) met our search criteria. Our search revealed a total of eight studies that experimentally manipulated laptop versus longhand note taking, assessed immediate quiz performance on the same day as exposure to the lecture, used video lecture material, measured and reported results for quiz performance, word count, and verbatim overlap, and studied undergraduates.

It is likely that our mini-meta-analyses do not include all unpublished attempts to replicate the original Study 1 by Mueller and Oppenheimer (2014). If a preponderance of unpublished attempts to replicate the original study have found that longhand note-taking prompts superior quiz performance compared to laptop note-taking, then our mini-metaanalytic estimates for quiz performance are biased toward zero. If, on the other hand, a preponderance of unpublished attempts to replicate found null results (consistent with the file drawer problem), then including them would have little effect on our mini-meta-analytic estimates for quiz performance; they are already close to zero.

\section{Correlations Between Effect Sizes from the Mini-Meta-Analyses}

One question is whether note-taking condition effects on notes content variables are correlated with note-taking condition effects on quiz performance variables at the study level. As shown in Figure A1, generated using the car package in R (Fox \& Weisberg, 
2011; Fox et al., 2018), lower laptop superiority (i.e., less negative values) for both word count, $r=.83,95 \%$ CI $[.21, .97]$, and verbatim overlap, $r=.80,95 \%$ CI $[.11, .97]$, were associated with greater longhand superiority (i.e., more positive values) for factual quiz performance. The confidence intervals indicated that the data were compatible with a wide range of positive associations from small to very large.

Associations between note-taking condition effects on conceptual and total quiz performance and note-taking condition effects on words, $r=-.16,95 \%$ CI $[-.81, .68]$ and $r=.00,95 \%$ CI $[-.70, .71]$, respectively, and verbatim overlap, $r=.06,95 \%$ CI $[-.73, .78]$ and $r=.35,95 \%$ CI $[-.47, .85]$, respectively, were much smaller. Moreover, the confidence intervals indicated that the data were compatible with a wide range of negative and positive associations, including a nil association.

We also found that studies showing laptop superiority for word count tended to show laptop superiority for verbatim overlap, $r=.82,95 \%$ CI $[.26, .97]$; the confidence interval indicated that the data were compatible with a wide range of positive associations from small to very large. Studies showing laptop or longhand superiority for factual quiz performance tended to show parallel laptop or longhand superiority for conceptual quiz performance, $r=.31,95 \%$ CI $[-.58, .86]$; the confidence interval indicated that the data were compatible with a wide range of negative and positive associations, including a nil association.

\section{Additional Exploratory Analyses}

Bayes Factor (BF) tests. We used logic and code provided by Verhagen and Wagenmakers (2014) to conduct Jeffreys-Zellner-Siow (JZS) BF and replication BF tests supported by the BayesFactor (Morey \& Rouder, 2018), MCMC (Martin, Quinn, \& Park, 2011), and polspline (Kooperberg, 2018) packages. According to Verhagen and Wagenmakers (2014), the replication BF test addresses the question, "Is the effect from the replication attempt comparable to what was found before, or is it absent?" By setting a prior based on the original effect, the replication BF test quantifies relative support for the replication hypothesis (i.e., that the replication study effect size is similar to the original study effect size) versus the null hypothesis (i.e., that the replication study effect size is zero). We also report a JZS BF test to evaluate evidence for the note-taking condition effect in the original and the replication studies separately. For these tests, we used a standard two-sided Cauchy $(0$, 1) distribution as the prior; results indicate relative support for the presence or absence of a note-taking condition effect. For the replication and JZS BF tests, numbers greater than 3 or less than 0.33 represent nonanecdotal support for the replication/alternative and null hypotheses, respectively.

For factual-recall performance, the original study revealed nonanecdotal support favoring the null hypothesis over the alternative hypothesis, $\mathrm{JZS} \mathrm{BF}_{10}=0.19$. The current replication study also revealed nonanecdotal support favoring the null hypothesis over the alternative hypothesis, $\mathrm{JZS}_{\mathrm{S}} \mathrm{BF}_{10}=0.15$. The replication hypothesis was somewhat less likely than the null hypothesis, replication $\mathrm{BF}_{10}=0.60$, although support for the null hypothesis was anecdotal.

For conceptual-application performance, the original study surprisingly revealed anec- 


\section{Scatter plot matrix of associations between effect sizes}
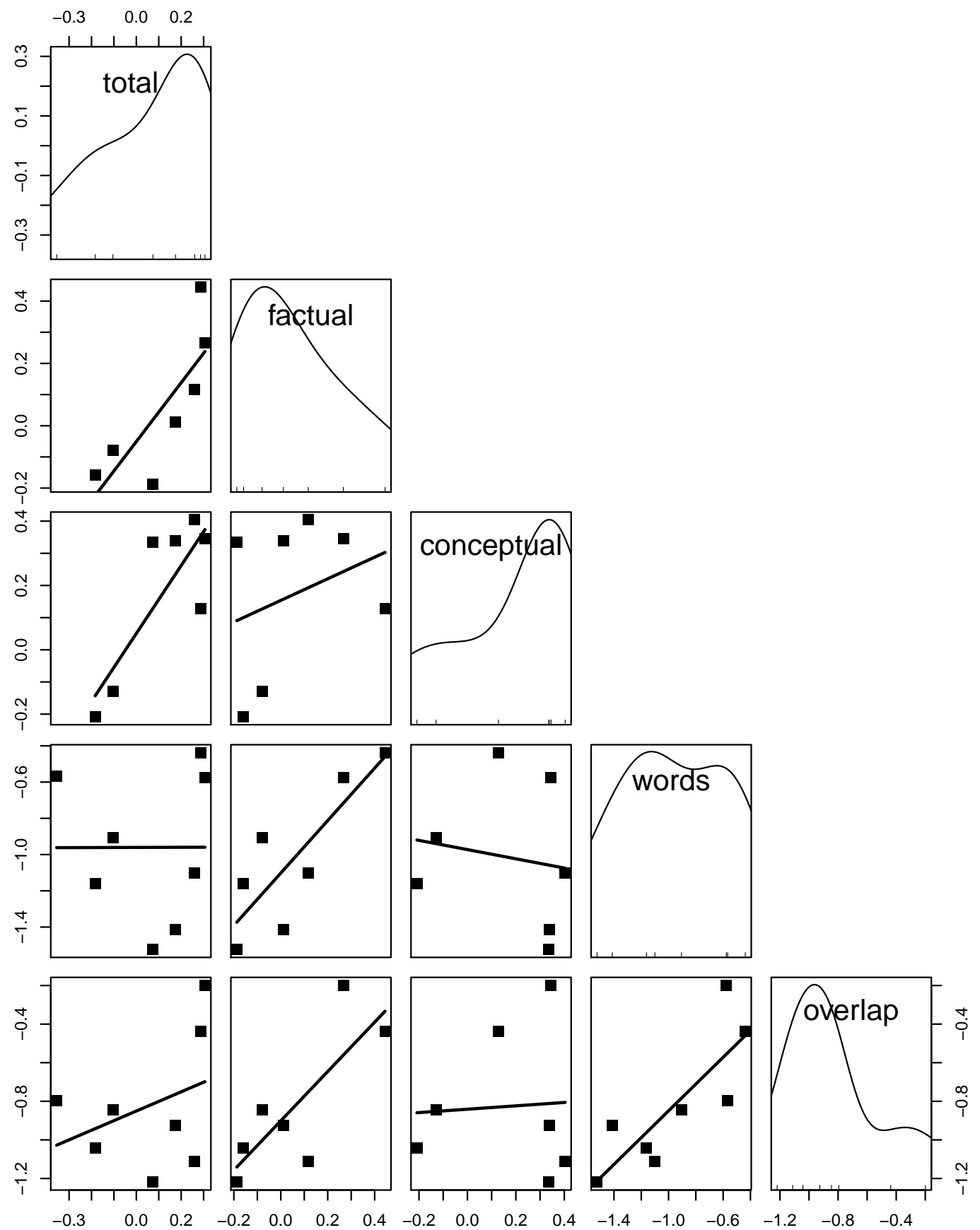

Figure A1. Scatter plot matrix showing associations between longhand-laptop effect sizes for the primary measured variables across 7-8 studies off the diagonal. Shown on the diagonal are kernel density estimates across effect sizes for each variable. 
dotal support favoring the null hypothesis over the alternative hypothesis, $\mathrm{JZS} \mathrm{BF}_{10}=0.45$. The current replication study revealed nonanecdotal support for the null hypothesis over the alternative hypothesis, $\mathrm{JZS}_{\mathrm{BF}_{10}}=0.17$. The replication hypothesis was much less likely than the null hypothesis, replication $\mathrm{BF}_{10}=0.22$.

By contrast, for both word count and verbatim overlap, the original study revealed nonanecdotal support favoring the alternative hypothesis over the null hypothesis, $\mathrm{JZS} \mathrm{BF}_{10}$ $=32,503.15$ and $\mathrm{JZS} \mathrm{BF}_{10}=59.80$, respectively. The current replication study also revealed nonanecdotal support favoring the alternative hypothesis over the null hypothesis, JZS BF 10 $=21,267.55$ and $\mathrm{JZS} \mathrm{BF}_{10}=7,882.39$, respectively. The replication hypothesis was much more likely than the null hypothesis, replication $\mathrm{BF}_{10}=42,555.42$ and replication $\mathrm{BF}_{10}=$ $51,402.76$, respectively.

Treating Item Type as a Factor. In the original article, the authors distinguished between factual and conceptual item types because, "Previous studies have shown that detriments due to verbatim note-taking are more prominent for conceptual than for factual items (e.g., Bretzing \& Kulhavy, 1979)" (Mueller \& Oppenheimer, 2014, p. 1160). Analyses focused separately on factual-recall and conceptual-application quiz performance, perhaps because there were more quiz items representing factual than conceptual information for some lectures.

However, we might have had greater sensitivity to detect true effects, if present, by examining performance across item type given a potential increase in measurement reliability. Moreover, strong inferences about differential effects of note-taking condition on these different item types require an interaction between condition and item type. We, thus, conducted mixed-effect analyses of variance of both the original Study 1 data and this replication using the afex package (Singmann, Bolker, Westfall, Aust, \& Ben-Shachar, 2019). We examined fixed effects of note-taking condition, item type, and their interaction, and random effects of lecture and participant; the dependent variable was quiz performance ( $\mathrm{Z}$ index scores). For those who might argue that lecture should be a fixed effect, we also conducted analyses of variance with fixed effects of note-taking condition, item type, lecture, and their interactions.

In the original Study 1, based on the analysis with random effects, neither the main effect of note-taking condition, $F(1,63)=1.01, M S E=0.99, p=.319, \hat{\eta}_{G}^{2}=.008$, nor its interaction with item type, $F(1,63)=0.87, M S E=1.01, p=.355, \hat{\eta}_{G}^{2}=.007$, explained significant variation in quiz performance. The same was true in the all fixed-effects analysis; neither the main effect of note-taking condition, $F(1,55)=1.45, M S E=0.98, p=.234$, $\hat{\eta}_{G}^{2}=.014$, nor its interaction with item type, $F(1,55)=0.78, M S E=0.87, p=.381$, $\hat{\eta}_{G}^{2}=.007$, explained significant variation in quiz performance. There also was no threeway interaction, $F(4,55)=0.60, M S E=0.87, p=.662, \hat{\eta}_{G}^{2}=.020$.

Similarly, in our replication, neither the main effect of note-taking condition, $F(1,140)=0.61, M S E=1.26, p=.436, \hat{\eta}_{G}^{2}=.003$, nor its interaction with item type, $F(1,140)=0.05, M S E=0.75, p=.825, \hat{\eta}_{G}^{2}=.000$, explained significant variation in quiz performance. The same was true in the all fixed-effects analysis; neither the main effect of note-taking condition, $F(1,132)=0.74, M S E=1.00, p=.390, \hat{\eta}_{G}^{2}=.004$, nor its interaction with item type, $F(1,132)=0.17, M S E=0.54, p=.682, \hat{\eta}_{G}^{2}=.000$, ex- 
plained significant variation in quiz performance. There also was no three-way interaction, $F(4,132)=0.84, M S E=0.54, p=.500, \hat{\eta}_{G}^{2}=.009$.

Continuous Predictors of Quiz Performance. In the original study, higher word count and lower verbatim overlap were associated with better quiz performance, and these two variables mediated the effect of note-taking condition on quiz performance. Moreover, it was possible that accounting for variability in quiz performance due to these and other covariates of interest might have revealed hypothesized effects of note-taking condition. Thus, in a set of linear mixed-effect regressions, we examined associations between word count, verbatim overlap, and other covariates - knowledge related to the lecture topic, beliefs about whether taking notes using a laptop or notebook is better for learning, need for cognition, typing speed and accuracy, or distraction duration - and quiz performance in our direct replication. We examined proportion correct for these analyses to facilitate interpretation in units of everyday interest. We conducted these analyses using the lme 4 package (Bates, Maechler, Bolker, \& Walker, 2015) and generated tables using the stargazer package (Hlavac, 2018).

In model 1, we entered condition (treatment contrast: laptop [0], longhand [1]), item type (sum contrast: factual [1], conceptual [-1]), and their interaction as fixed effects. There were random intercepts for lectures and participants, and random slopes for item type across lectures. (Models including a random slope for condition across lectures did not converge; fits were singular.) In model 2 , we added 8 centered covariates to model 1 . In model 3 , we repeated model 2 excluding participants identified as influential using the influence.ME package (Nieuwenhuis, Te Grotenhuis, \& Pelzer, 2012). In model 4, we repeated model 2, this time including word count and verbatim overlap, the only two statistically significant covariates. Finally, in model 5, we repeated model 4 excluding participants identified as influential again using influence.ME.

Table A2 summarizes all five models. According to Akaike and Bayesian information criteria, model 5 provided the best fit to the data. Consistent with the original study, higher word count was associated with better quiz performance in all models. Also consistent with the original study, higher verbatim overlap was significantly associated with lower quiz performance in models 2-4. In model 5, however, the magnitude of the association between verbatim overlap and quiz performance was reduced by half and no longer statistically significant. Figure A2 shows the linear associations between the notes content variables and quiz performance from models 4 and 5, generated using the effects package (Fox \& Weisberg, 2018).

Model 5 raises doubt about the robustness of the negative association between verbatim overlap and quiz performance, especially since it was the best-fitting model. To investigate further, we conducted a robust version of model 4 using the robustlmm package (Koller, 2016), which applies robustness weights based on a random effects contamination model to reduce influence. In the robust version of model 4 , the word count association was again positive, $b=0.05, S E=0.014, t=3.536$, and the verbatim overlap association was again negative, $b=-0.045, S E=0.014, t=-3.185$; both estimates were similar in magnitude to estimates derived from models 2-4. Although robustlmm does not provide $p$ values for significance testing, both $t$ values were larger than $|1.96|$, which suggests 
Table A2

Linear mixed-effect regression models for quiz performance (proportion correct) with covariates

\begin{tabular}{|c|c|c|c|c|c|}
\hline & $\begin{array}{c}\text { Item Type as Factor } \\
(1)\end{array}$ & $\begin{array}{c}(1)+8 \text { Covariates } \\
(2)\end{array}$ & $\begin{array}{c}(2) \text { - Influential } \\
(3)\end{array}$ & $\begin{array}{c}(2)-6 \text { Covariates } \\
(4)\end{array}$ & $\begin{array}{c}(4) \text { - Influential } \\
(5)\end{array}$ \\
\hline $\begin{array}{l}\text { Intercept (laptop }=0 \text { ) } \\
\text { condition (longhand }=1 \text { ) } \\
\text { item type } \\
\text { word count } \\
\text { verbatim overlap } \\
\text { related knowledge } \\
\text { better laptop or notebook } \\
\text { need for cognition } \\
\text { typing accuracy } \\
\text { typing speed } \\
\text { distraction duration }\end{array}$ & $\begin{array}{l}.681^{* * *}(.033) \\
-.017(.025) \\
-.056(.038)\end{array}$ & $\begin{array}{l}.671^{* * *}(.030) \\
-.007(.029) \\
-.052(.041) \\
.056^{* * *}(.016) \\
-.046^{* *}(.017) \\
.027(.014) \\
.022(.013) \\
-.005(.013) \\
-.003(.014) \\
-.006(.016) \\
-.013(.014)\end{array}$ & $\begin{array}{l}.675^{* * *}(.030) \\
.025(.025) \\
-.052(.041) \\
.053^{* * *}(.014) \\
-.039^{*}(.016) \\
.022(.012) \\
.020(.012) \\
-.010(.011) \\
-.013(.012) \\
-.005(.014) \\
-.019(.012)\end{array}$ & $\begin{array}{l}.680^{* * *}(.029) \\
-.015(.028) \\
-.054(.038) \\
.054^{* * *}(.016) \\
-.047^{* *}(.015)\end{array}$ & $\begin{array}{c}.683^{* * *}(.029) \\
.006(.026) \\
-.052(.038) \\
.048^{* *}(.016) \\
-.023(.016)\end{array}$ \\
\hline condition*item type & $.010(.018)$ & $.002(.019)$ & $.004(.020)$ & $.008(.018)$ & $.006(.018)$ \\
\hline $\begin{array}{l}\text { Observations } \\
\text { Log Likelihood } \\
\text { Akaike Inf. Crit. } \\
\text { Bayesian Inf. Crit. }\end{array}$ & $\begin{array}{c}284 \\
74.350 \\
-130.700 \\
-97.860 \\
\end{array}$ & $\begin{array}{c}262 \\
75.920 \\
-117.800 \\
-57.170 \\
\end{array}$ & $\begin{array}{c}246 \\
90.140 \\
-146.300 \\
-86.690 \\
\end{array}$ & $\begin{array}{c}280 \\
80.100 \\
-138.200 \\
-98.220 \\
\end{array}$ & $\begin{array}{c}268 \\
89.220 \\
-156.400 \\
-116.900 \\
\end{array}$ \\
\hline
\end{tabular}

Note:

${ }^{*} \mathrm{p}<0.05 ;{ }^{* *} \mathrm{p}<0.01 ;{ }^{* * *} \mathrm{p}<0.001$ 

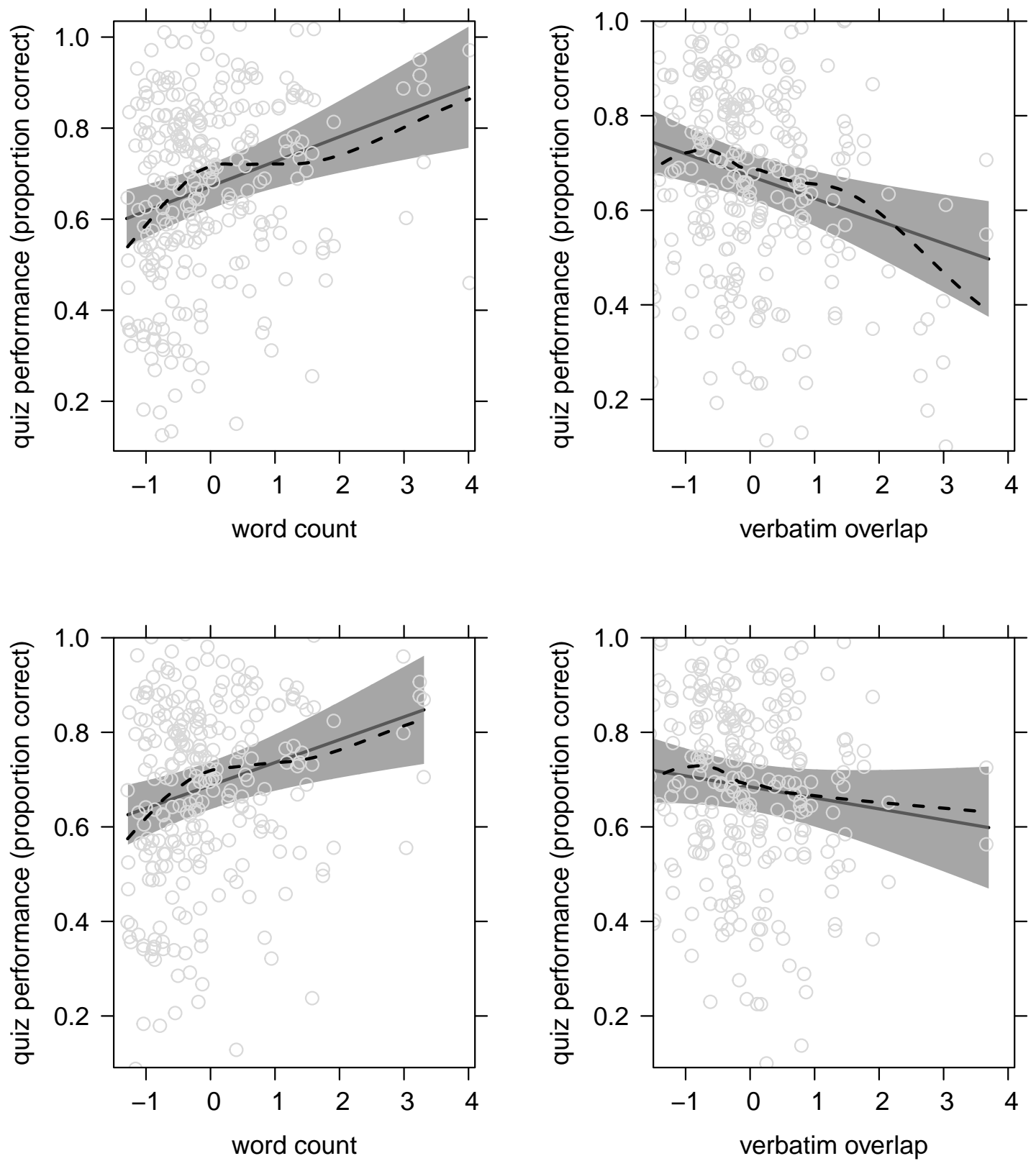

Figure A2. Scatter plot of partial residuals (open light gray points) depicting associations between notes content variables on the $\mathrm{X}$-axes (word count and verbatim overlap) and quiz performance on the Y-axes. These reflect associations from LMER models 4 (top row; all observations) and 5 (bottom row; excluding influential observations). The solid black prediction lines depict the linear associations; they are surrounded by $95 \%$ confidence bands in dark gray. The dashed black lines depict loess nonparametric-regression smoothing of the observations. 
statistical significance.

In sum, whereas the best-fitting linear mixed-effect regression suggested that higher word count but not lower verbatim overlap was associated with better quiz performance, a robust version of that analysis supported both associations. There is, thus, some ambiguity as to the extent of negative association between verbatim overlap and quiz performance in this study.

Of note, accounting for variability in performance attributable to the covariates did not reveal the hypothesized effect of note-taking condition on its own or in interaction with item type. Similar conclusions emerge from an analysis examining the fixed unique and interactive effects of condition, item type, and lecture with the same covariates and with random intercepts for participants (not shown). Associations between other covariates and quiz performance were generally closer to zero and not statistically significant, as reflected in models 2 and 3.

\section{Difference in Laptop versus Longhand Note Taking Preferences}

It's possible that note-taking medium preferences among original and replication study participants may have differed. To evaluate that possibility, we compared responses to the question, "Do you normally take notes in class on your laptop or in a notebook? Why?". We obtained the original authors' coded responses from the SPSS Study_1_Upload_Data.sav file stored at https://osf.io/4psyk. They categorized responses into four categories, "laptop", "notebook", "it depends", or 0, which we interpret as "other," meaning the response didn't fall into one of the first three categories or was missing. We coded replication responses to that question into these same categories.

The distribution of note-taking medium preferences varied by study, $\chi^{2}(3, n=207)=$ $28.45, p<.001$. In the original study, proportionally more participants said they typically use a laptop $(50.77 \%)$ than a notebook $(30.77 \%)$ to take notes during class, and $15.38 \%$ said it depends. In our replication study, proportionally more participants said they typically use a notebook $(53.52 \%)$ than a laptop $(15.49 \%)$ to take notes during class, and $26.06 \%$ said it depends.

The extent to which original and replication study participants believed that taking notes with a laptop or notebook is better for learning varied by study too, $\Delta M=-0.76,95 \%$ CI $[-1.27,-0.25], t(108.19)=-2.97, p=.004$. Specifically, in both studies, participants responded to the question, "In general, do you think it is better for learning purposes to take notes on a laptop or in a notebook?" on a scale from 1, Laptop significantly better, to 9, Notebook significantly better. In the original study, participants on average believed that laptop and notebook note taking were equally good for learning; the mean hovered near the scale midpoint, $M=4.48,95 \%$ CI $[4.03,4.92], S D=1.80$. In our replication study, participants on average believed that taking notes in a notebook was slightly better for learning than taking notes with a laptop; the mean fell to the right of the scale midpoint, $M=5.24,95 \%$ CI $[4.99,5.49], S D=1.53$.

These results indicate that note-taking medium preferences are different in the two samples. Our replication participants were more likely to say they generally took class notes 
longhand whereas original study participants were more likely to say they generally took notes using a laptop. Our replication participants also believed, on average, that taking notes in a notebook was better for learning whereas original study participants believed, on average, that there wasn't much of a difference.

\section{Session Information}

Following is the output of R's sessionInfo() command, which reveals the information necessary to ensure analytic reproducibility of our work.

R version 4.0.2 (2020-06-22) Platform: x86_64-w64-mingw32/x64 (64-bit) Running under: Windows 10 x64 (build 17134)

Matrix products: default

Random number generation: RNG: Mersenne-Twister Normal: Inversion Sample: Rounding

locale: $\quad[1] \quad$ LC_COLLATE=English_United States.1252 [2]

LC_CTYPE $=$ English_United States. 1252

[3] LC_MONETARY=English_United States.1252 [4] LC_NUMERIC $=\mathrm{C}$

[5] LC_TIME=English_United States. 1252

attached base packages: [1] stats graphics grDevices utils datasets methods base

other attached packages: [1] knitr_1.29 haven_2.3.1

[3] influence.ME_0.9-9 robustlmm_2.3

[5] effects_4.1-4 afex_0.27-2

[7] dplyr_1.0.0 userfriendlyscience_0.7.2 [9] car_3.0-8 carData_3.0-4

[11] stargazer_5.2.2 tidyr_1.1.0

[13] MBESS_4.7.0 metafor_2.4-0

[15] lmerTest_3.1-2 lme4_1.1-23

[17] effsize_0.8.0 polspline_1.1.19

[19] MCMCpack_1.4-8 MASS_7.3-51.6

[21] pwr_1.3-0 TOSTER_0.3.4

[23] compute.es_0.2-5 yarrr_0.1.5

[25] circlize_0.4.10 BayesFactor_0.9.12-4.2

[27] Matrix_1.2-18 coda_0.19-3

[29] jpeg_0.1-8.1 psych_1.9.12.31

[31] papaja_0.1.0.9997

loaded via a namespace (and not attached): [1] readxl_1.3.1 plyr_1.8.6 GPArotation_2014.11-1 [4] splines_4.0.2 ufs_0.3.1 ggplot2_3.3.2

[7] TH.data_1.0-10 digest_0.6.25 SuppDists_1.1-9.5

[10] htmltools_0.5.0 viridis_0.5.1 magrittr_1.5

[13] openxlsx_4.1.5 readr_1.3.1 matrixStats_0.56.0

[16] sandwich_2.5-1 colorspace_1.4-1 ggrepel_0.8.2

[19] fastGHQuad_1.0 mitools_2.4 BiasedUrn_1.07

[22] xfun_0.15 crayon_1.3.4 jsonlite_1.7.0 
[25] SCRT_1.3.1 survival_3.1-12 zoo_1.8-8

[28] glue_1.4.1 gtable_0.3.0 emmeans_1.4.8

[31] MatrixModels_0.4-1 shape_1.4.4 DEoptimR_1.0-8

[34] abind_1.4-5 SparseM_1.78 scales_1.1.1

[37] mvtnorm_1.1-1 DBI_1.1.0 GGally_2.0.0

[40] data.tree_0.7.11 Rcpp_1.0.5 viridisLite_0.3.0

[43] xtable_1.8-4 tmvnsim_1.0-2 foreign_0.8-80

[46] stats4_4.0.2 survey_4.0 htmlwidgets_1.5.1

[49] DiagrammeR_1.0.6.1 RColorBrewer_1.1-2 lavaan_0.6-6

[52] ellipsis_0.3.1 farver_2.0.3 pkgconfig_2.0.3

[55] reshape_0.8.8 XML_3.99-0.4 nnet_7.3-14

[58] labeling_0.3 tidyselect_1.1.0 rlang_0.4.7

[61] reshape2_1.4.4 munsell_0.5.0 cellranger_1.1.0

[64] tools_4.0.2 visNetwork_2.0.9 generics_0.0.2

[67] ggridges_0.5.2 evaluate_0.14 stringr_1.4.0

[70] yaml_2.2.1 mcmc_0.9-7 robustbase_0.93-6

[73] zip_2.0.4 pander_0.6.3 purrr_0.3.4

[76] pbapply_1.4-2 nlme_3.1-148 quantreg_5.61

[79] compiler_4.0.2 curl_4.3 tibble_3.0.3

[82] statmod_1.4.34 pbivnorm_0.6.0 stringi_1.4.6

[85] highr_0.8 forcats_0.5.0 lattice_0.20-41

[88] nloptr_1.2.2.2 vetrs_0.3.2 pillar_1.4.6

[91] lifecycle_0.2.0 GlobalOptions_0.1.2 estimability_1.3

[94] data.table_1.12.8 conquer_1.0.1 R6_2.4.1

[97] bookdown_0.20 gridExtra_2.3 rio_0.5.16

[100] codetools_0.2-16 boot_1.3-25 gtools_3.8.2

[103] minpack.lm_1.2-1 mnormt_2.0.1 multcomp_1.4-13

[106] diptest_0.75-7 parallel_4.0.2 hms_0.5.3

[109] grid_4.0.2 minqa_1.2.4 rmarkdown_2.3

[112] numDeriv_2016.8-1.1

Bates, D., Maechler, M., Bolker, B., \& Walker, S. (2015). Fitting linear mixedeffects models using lme4. Journal of Statistical Software, 67(1), 1-48. https: //doi.org/10.18637/jss.v067.i01

Fox, J., \& Weisberg, S. (2011). An $R$ companion to applied regression (Second). Thousand Oaks CA: Sage. Retrieved from http://socserv.socsci.mcmaster.ca/ jfox/Books/Companion

Fox, J., \& Weisberg, S. (2018). Visualizing fit and lack of fit in complex regression models with predictor effect plots and partial residuals. Journal of Statistical Software, 87(9), 1-27. https://doi.org/10.18637/jss.v087.i09

Fox, J., Weisberg, S., \& Price, B. (2018). CarData: Companion to applied regression data sets. Retrieved from https://CRAN.R-project.org/package=carData

Hlavac, M. (2018). Stargazer: Well-formatted regression and summary statistics tables. Bratislava, Slovakia: Central European Labour Studies Institute (CELSI). 
Retrieved from https://CRAN.R-project.org/package=stargazer

Koller, M. (2016). robustlmm: An R package for robust estimation of linear mixedeffects models. Journal of Statistical Software, 75(6), 1-24. https://doi.org/10. $18637 /$ jss.v075.i06

Kooperberg, C. (2018). Polspline: Polynomial spline routines. Retrieved from https://CRAN.R-project.org/package=polspline

Martin, A. D., Quinn, K. M., \& Park, J. H. (2011). MCMCpack: Markov chain monte carlo in R. Journal of Statistical Software, 42(9), 22. Retrieved from http://www.jstatsoft.org/v42/i09/

Morey, R. D., \& Rouder, J. N. (2018). BayesFactor: Computation of bayes factors for common designs. Retrieved from https://CRAN.R-project.org/package= BayesFactor

Nieuwenhuis, R., Te Grotenhuis, M., \& Pelzer, B. (2012). Influence.ME: Tools for detecting influential data in mixed effects models. $R$ Journal, 4(2), 38-47.

Peters, G.-J. Y. (2017). Diamond plots: A tutorial to introduce a visualisation tool that facilitates interpretation and comparison of multiple sample estimates while respecting their inaccuracy. PsyArXiv. Retrieved from https://psyarxiv.com/ fzh6c

Petty, R. E., Cacioppo, J. T., \& Kao, C. F. (1984). The efficient assessment of need for cognition. Journal of Personality Assessment, 48(3), 306-307.

Revelle, W. (2018). Psych: Procedures for psychological, psychometric, and personality research. Evanston, Illinois: Northwestern University. Retrieved from https://CRAN.R-project.org/package $=$ psych

Shrout, P. E., \& Fleiss, J. L. (1979). Intraclass correlations: Uses in assessing rater reliability. Psychological Bulletin, 86(2), 420.

Singmann, H., Bolker, B., Westfall, J., Aust, F., \& Ben-Shachar, M. S. (2019). Afex: Analysis of factorial experiments. Retrieved from https://CRAN.R-project.org/ package $=$ afex

Verhagen, J., \& Wagenmakers, E.-J. (2014). Bayesian tests to quantify the result of a replication attempt. Journal of Experimental Psychology: General, 143(4), $1457-1475$. 